\title{
Estimation of fracture compliance from attenuation and velocity analysis of full-waveform sonic log data
} Nicolás D. Barbosa ${ }^{1 *}$, Eva Caspari ${ }^{1}$, J. Germán Rubino ${ }^{2}$, Andrew Greenwood $^{1}$, Ludovic Baron ${ }^{1}$, and Klaus Holliger ${ }^{1,3}$

* Now at University of Geneva, Geneva, Switzerland

Manuscript accepted for publication in J. Geophys. Res.: Solid Earth

${ }^{1}$ Applied and Environmental Geophysics

Group, Institute of Earth Sciences,

University of Lausanne, Lausanne,

Switzerland

${ }^{2}$ CONICET, Centro Atómico Bariloche -

CNEA, San Carlos de Bariloche, Argentina

${ }^{3}$ School of Earth Sciences, Zhejiang

University, Hangzhou, China 


\section{Abstract.}

${ }_{4} \quad$ In fractured rocks, the amplitudes of propagating seismic waves decay due

${ }_{5}$ to various mechanisms, such as geometrical spreading, solid friction, displace-

6 ment of pore fluid relative to the solid frame, and transmission losses due

7 to energy conversion to reflected and transmitted waves at the fracture in-

s terfaces. In this work, we characterize the mechanical properties of individ-

9 ual fractures from P-wave velocity changes and transmission losses inferred

${ }_{10}$ from static full-waveform sonic (FWS) log data. The methodology is vali-

${ }_{11}$ dated using synthetic FWS logs and applied to data acquired in a borehole

${ }_{12}$ penetrating multiple fractures embedded in a granodioritic rock. To extract

${ }_{13}$ the transmission losses from attenuation estimates, we remove the contribu-

${ }_{14}$ tions associated with other loss mechanisms. The geometrical spreading cor-

${ }_{15}$ rection is inferred from a joint analysis of numerical simulations that emu-

${ }_{16}$ late the borehole environment and the redundancy of attenuation contribu-

${ }_{17}$ tions other than geometrical spreading in multiple acquisitions with differ-

${ }_{18}$ ent source-receiver spacing configurations. The intrinsic background atten-

${ }_{19}$ uation is estimated from measurements acquired in the intact zones. In the

${ }_{20}$ fractured zones, the variations with respect to the background attenuation

${ }_{21}$ are attributed to transmission losses. Once we have estimated the transmis-

${ }_{22}$ sion losses associated with a given fracture, we compute the transmission co-

${ }_{23}$ efficient, which, on the basis of the linear slip theory, can then be related to

${ }_{24}$ the mechanical normal compliance of the fracture. Our results indicate that

25 the estimated mechanical normal compliance ranges from $1 \times 10^{-13} \mathrm{~m} / \mathrm{Pa}$ to 
${ }_{26} 1 \times 10^{-12} \mathrm{~m} / \mathrm{Pa}$, which, for the size of the considered fractures, is consistent

27 with the experimental evidence available. 


\section{Introduction}

Fractures have a predominant influence on the mechanical behavior of a rock mass as they provide planes of weakness which decrease the overall stiffness of an otherwise intact

so medium [e.g. Schoenberg and Douma, 1988]. Fractures also often constitute the major ${ }_{31}$ conduits through which fluids can flow. This makes their characterization an important task for many important applications, such as, for example, the development of oil and

33 gas reservoirs, the production of geothermal energy, the understanding and prediction of

34 the performance of underground radioactive-waste repositories, and the geological storage

35 of $\mathrm{CO}_{2}$ [Zimmerman and Main, 2004; Bakku et al., 2013]. Given that seismic waves prop-

${ }_{36}$ agating through fractured rocks are known to be slowed down and attenuated, seismic

${ }_{37}$ methods are valuable for characterizing the hydromechanical behavior of these environ-

38 ments.

39 The effects of fractures on seismic wave propagation strongly depend on the relation

40 between the characteristic size of the fractures, their separation, and the prevailing seismic

${ }_{41}$ wavelengths [e.g. Fang et al., 2017]. Many analytical and numerical models have been

${ }_{42}$ proposed to study seismic wave propagation in rocks containing cracks or fractures that

${ }_{43}$ are much smaller than the wavelengths [e.g. Hudson, 1980; Schoenberg and Douma, 1988;

${ }_{44}$ Chapman, 2003; Gurevich, 2003; Rubino et al., 2013; Sil, 2013]. In that case, an effective

45 stiffness tensor, which, in the most general case, is anisotropic with complex-valued and

${ }_{46}$ frequency-dependent elements, allows for describing seismic wave propagation through the

${ }_{47}$ fractured medium. However, when the distance between fractures as well as their size are

${ }_{48}$ large relative to the seismic wavelength, effective medium approaches are not appropriate 
${ }_{49}$ [Schoenberg and Douma, 1988]. Instead fractures must be treated as distinct features.

50 Such sparsely spaced individual fractures can have significant effects on the amplitudes and

${ }_{51}$ velocities of seismic waves as shown through laboratory experiments [Pyrak-Nolte et al.,

${ }_{52}$ 1990; Pyrak-Nolte and Nolte, 1992; Lubbe et al., 2008], numerical simulations [Barbosa

${ }_{53}$ et al., 2016], field seismic measurements [Worthington and Hudson, 2000], or combined

${ }_{54}$ approaches [Morris et al., 1964; Minato and Ghose, 2016]. The corresponding evidence

${ }_{55}$ suggests that the two most likely mechanisms for explaining the effects of fluid-saturated

${ }_{56}$ single fractures on seismic wave propagation are wave-induced fluid flow (WIFF) and

${ }_{57}$ energy conversion to reflected and transmitted waves [Baird et al., 2013].

${ }_{58}$ An inherent problem associated with the interpretation of seismic attenuation and veloc-

${ }_{59}$ ity dispersion in terms of mechanical and hydraulic properties is the necessity to separate

${ }_{60}$ the contributions of the various extrinsic (e.g., scattering, geometrical spreading) and in-

${ }_{61}$ trinsic (e.g., solid friction, WIFF) physical mechanisms involved. In the particular case

${ }_{62}$ of sonic wave propagation in a borehole, this issue has been adressed for layered for-

${ }_{63}$ mations [Sams, 1991; Parra et al., 2007], lithologically and hydraulically heterogeneous

${ }_{64}$ formations [Sun et al., 2000], gas hydrate-bearing sediments [Guerin and Goldberg, 2002],

${ }_{65}$ water-saturated alluvial sediments [Milani et al., 2015], and partially saturated gas shales

${ }_{66}$ [Qi et al., 2017], among others. For all these environments, it has been found that a

${ }_{67}$ critical aspect for extracting information on the intrinsic attenuation of the probed for-

${ }_{68}$ mation is to adequately compensate for the effects of geometrical spreading. Indeed, Sams

69 [1991] found negative $Q$-values in a sequence of weakly consolidated turbiditic sediments,

70 which he attributed to inaccurate compensation for geometrical spreading. Milani et al.

${ }_{71}[2015]$ pointed out that the inconsistency between the sonic P-wave velocity dispersion 
72 and attenuation estimates of Baron and Holliger [2010] was due to an incorrect estima-

${ }_{73}$ tion of the geometrical spreading correction. In the case of fractured environments, only

${ }_{74}$ qualitative correlations between anomalously high sonic attenuation and the presence of

75 fractures have been reported [e.g. Sun et al., 2000]. Hence, identifying and separating

${ }_{76}$ the effects related to the different contributions to the energy dissipation of sonic waves

77 in the presence of fractures is the first step for a quantitative interpretation of fracture

${ }_{78}$ properties.

79 So far, quantitative fracture characterization from seismic data in general and from

so sonic log data in particular has been mostly limited to the modelling of the decrease in

${ }_{81}$ the phase velocity due to the presence of fractures [Moos and Zoback, 1983; Lubbe and

${ }_{82}$ Worthington, 2006; Prioul and Jocker, 2009]. In this context, fractures are often charac-

вз terized based on the linear slip model, in which fractures are represented as boundaries

${ }_{84}$ across which the seismic stress is continuous but the displacements are not. The link

${ }_{85}$ between the magnitude of the displacement discontinuity across the fracture and the im-

${ }_{86}$ posed seismic stress is given by the effective mechanical compliance of the fracture. From

${ }_{87}$ this effective property other fracture properties, such as, the aperture, the contact area

${ }_{88}$ distribution, the stress field, and the infill material of the voids between the fracture in-

${ }_{89}$ terfaces, can be inferred through different mechanical models [Hudson et al., 1996; Liu

${ }_{90}$ et al., 2000; Zimmerman and Main, 2004; Prioul and Jocker, 2009; Minato and Ghose,

${ }_{91}$ 2016]. Given that scattered seismic wave fields depend on the fracture compliance, the

${ }_{92}$ use of the reflection or transmission response of a fracture for its characterization is very

${ }_{93}$ common [e.g. Pyrak-Nolte et al., 1990; Yoshioka and Kikuchi, 1993; Minato and Ghose,

${ }_{94}$ 2016]. Exploiting this idea, fracture compliances have been extensively computed based 
95 on laboratory measurements on real and synthetic samples. However, almost all rock masses contain fractures on scales larger than that of core samples, with typical fracture

${ }_{97}$ spacings that range from tens of centimeters to tens of meters. Estimating fracture com-

${ }_{98}$ pliances from sonic log or seismic data can therefore not only provide information of larger

99 fractures, but also at in situ conditions, which can, for example, be directly utilized for planning and monitoring hydraulic fracturing operations [Bakku et al., 2013] or for assessing fracture hydraulic transmissivity [Pyrak-Nolte and Morris, 2000; Rutqvist, 2015; Kang et al., 2016]. Moreover, given that a medium containing a large number of small cracks or a few large fractures can yield the same effective anisotropy [Schoenberg and Douma, 1988], unraveling the relation between the size of fractures and their mechanical compliance may help to constrain the interpretation of seismic anisotropy. Despite its 
offset between the source and the first receiver is $182.8 \mathrm{~cm}$ (6 ft) (Fig. 1). In the following, we refer to the tool configurations with offsets of $91.4 \mathrm{~cm}$ and $182.8 \mathrm{~cm}$ between the first receiver and the source as "short" and "long", respectively. The temporal sampling rates were 4 and $8 \mu$ s for the short and long tool configurations, respectively.

In order to optimize the survey, we have used acoustic and optical televiewer images [Krietsch et al., 2018], which provide an estimation of the location, orientation, spacing and aperture of the features intersecting the borehole, to identify the zones characterized by the presence of individual fractures. As a result, static measurements were acquired at 33 different source depths using the short tool configuration. From this data set, we compute the velocity and attenuation as a function of depth and nominal source frequency. For the long tool configuration, only 6 source positions were recorded.

Due to the discontinuous depth sampling of the static FWS data, we have separated the data set into three subsets depending on the borehole section in which the measurements were taken. These sections are referred to as the upper, central, and lower sections. The upper section contains 9 short configuration and 6 long configuration measurement points with a spatial sampling of $60 \mathrm{~cm}$. The central section contains 13 measurement points for the short configuration with a spatial sampling rate of $60 \mathrm{~cm}$. And lastly, the lower section contains 11 short configuration measurements with a spatial sampling rate of $30 \mathrm{~cm}$. Table 1 summarizes the transmitter depths and spatial sampling for both tool configurations. Notice that, for the upper section, the receiver positions for the long and short configurations overlap. The long configuration measurements have been used to verify the robustness of the attenuation estimates and to obtain information on 


\section{Analysis of phase velocity and attenuation estimations from FWS data}

In this section, we first compute the sonic $\mathrm{P}$-wave phase velocity and attenuation profiles from FWS data. We then analyze the different contributions to the observed amplitude decay of the direct P-wave, with particular focus on quantifying those that are independent of the presence of fractures. This will allow us to extract the attenuation exclusively due to single fractures which, in turn, can be used to determine their mechanical compliances.

\subsection{Isolation of first-arriving $\mathrm{P}$-wave}

In order to perform an analysis of the P-wave phase velocity and attenuation, the measured arrivals must represent the critically refracted P-wave traveling along the borehole. We have separated such P-wave first-arrivals from later arrivals, such as, for example, Pwaves reflected at fractures, using a time window tapered at both ends with a half-cosine to reduce ringing effects. As the results can be quite sensitive to the time window utilized [Parra et al., 2007], we have tested two different time window lengths, comprising one and two cycles of the first P-wave arrival. Fig. 2 shows the static FWS data for the upper section of the borehole. The P-wave arrival is isolated using a window centered around the first (red line) and second (blue lines) cycles. For a time window centered at the first cycle of the first-arriving $\mathrm{P}$-wave, the amplitudes are expected to be less affected by later arrivals, and hence, provide more stable estimates of the $\mathrm{P}$-wave attenuation and phase velocity [Dasios et al., 2001]. However, for larger source-receiver offsets, such as for the long tool configuration, the signal-to-noise ratio of this first cycle might be poor. In that 


\subsection{Velocity analysis}

Following Molyneux and Schmitt [2000], we compute the P-wave phase velocity $v_{p}(\omega)$ from the difference of the phase spectra $\Delta \varphi$ of the signals recorded at two receivers as

$$
v_{p}(\omega)=\frac{\omega \Delta r}{\Delta \varphi(\omega)},
$$

where $\Delta r$ is the distance between the two receivers and $\omega$ the angular frequency. The phase difference is chosen so that the condition $\left|\Delta \varphi-\omega \Delta r / v_{0}\right|<\pi$ is fulfilled. Based on ultrasonic and continuous FWS measurements we used $v_{0}=5000 \mathrm{~m} / \mathrm{s}$.

Fig. 3 shows the $\mathrm{P}$-wave velocity for nominal source frequencies of 15 and $25 \mathrm{kHz}$ at depths corresponding to the three sections of the borehole. The frequency considered in each case corresponds to the peak of the amplitude spectrum at the first receiver. As $v_{p}(\omega)$ computed using Eq. 1 is the interval velocity between the two receivers, each step of the velocity profile corresponds to the distance between consecutive receivers $\operatorname{Rx}(i)$ and $\operatorname{Rx}(i+1)$. The black dots in Fig. 3 indicate the velocity computed from the phase difference between the signals at $\mathrm{Rx} 1$ and $\mathrm{Rx} 3$. Although it represents the interval velocity between Rx1 and Rx3, for illustration purposes, it has been plotted as a single value located at 
the depth of Rx2. As the receivers are equally spaced, it is equal to the harmonic average of the velocities measured between $\mathrm{Rx} 1$ and $\mathrm{Rx} 2$ and between $\mathrm{Rx} 2$ and $\mathrm{Rx} 3$. Given that the nominal source frequencies considered are quite close to each other and due to the uncertainties of the measurements, the computation of velocities at both frequencies is performed primarily to assure the reliability of the measurements rather than to quantify any velocity dispersion effects. That said, we observe that velocities at $15 \mathrm{kHz}$ are, in general, systematically lower than at $25 \mathrm{kHz}$.

Notice that Figs. 3a and b show continuous step velocity profiles as a result of combining the velocity estimations for pairs of receivers Rx1-Rx2 and Rx2-Rx3. Fig. 3c, on the other hand, shows the P-wave velocity profile considering only receivers $\mathrm{Rx} 2$ and $\mathrm{Rx} 3$, which is continuous due to the shorter spacing between source positions (Table 1). Overall, the $\mathrm{P}$-wave velocity in the intact background rock ranges between 5100 and $5200 \mathrm{~m} / \mathrm{s}$, which was found to be consistent with the velocities estimated from independent continuous FWS log data acquired in this borehole [Krietsch et al., 2018].

\subsubsection{Geological features}

In the following, we analyze the correlation between changes in the P-wave velocity and the presence of prominent geological features, such as fractures, ductile shear zones, and lamprophyre dykes, observed in the televiewer images (Fig. 3). The two lamprophyre dykes in the central section constitute the boundaries of a brittle overprint shear zone characterized by a higher fracture density compared to the rest of the rock mass [Wenning et al., 2018]. The majority of these brittle fractures are orientated parallel to the boundaries of the dykes [Jalali et al., 2017]. The shear zone located around $20 \mathrm{~m}$ depth in Fig. 3b has been hydraulically and mechanically characterized by Wenning et al. [2018] 
249 using core samples from a nearby borehole. In general, we observe a significant reduc-

${ }_{250}$ tion in $\mathrm{P}$-wave velocity in the presence of lamprophyre dykes. However, notice that in

${ }_{251}$ the central section, the dyke thicknesses are of the order of $10 \mathrm{~cm}$ and, hence, they are

${ }_{252}$ comparable to the prevailing wavelengths of $\sim 25 \mathrm{~cm}$ for a frequency of $\sim 20 \mathrm{kHz}$ and

${ }_{253}$ a representative $\mathrm{P}$-wave velocity of $\sim 5200 \mathrm{~m} / \mathrm{s}$ (Fig. 3). This, in turn, can affect the

${ }_{254}$ accuracy of the velocity estimations in the vicinity of these structures.

255 As illustrated by Fig. 3a, the intervals with fractures exhibit a less obvious correlation

${ }_{256}$ with velocity changes than dykes. In some cases, the presence of fractures does not

${ }_{257}$ produce a significant change in velocity compared to that of the surrounding background.

${ }_{258}$ As pointed out by Zimmerman and Main [2004], fractures may be open or may filled

${ }_{259}$ with (i) fault gouge that has been produced by shearing mechanisms, (ii) clay minerals,

${ }_{260}$ or (iii) mineral coatings that have been precipitated from pore fluids. Indeed, fractures

${ }_{261}$ corresponding to relatively high phase velocities are likely to be mineralized [Keusen

${ }_{262}$ et al., 1989; Majer et al., 1990]. Conversely, Figs. 3b) and c) show examples of fractures

${ }_{263}$ that produce a clear decrease in the P-wave velocity, thus acting as planes of mechanical

${ }_{264}$ weakness. Fractures allowing for enhanced mechanical deformation are also expected to

265 be more hydraulically open [e.g. Pyrak-Nolte and Nolte, 2016].

\subsection{Attenuation analysis}

${ }_{266}$ Using the $\mathrm{P}$-wave velocity profile and its correlation with the geological features observed ${ }_{267}$ in the televiewer images, we can identify zones where physical property contrasts may potentially influence seismic wave attenuation. In the following, we first describe the spectral ratio method employed to compute attenuation which is commonly used for

270 both laboratory and field measurements [e.g. Cheng et al., 1982; Pyrak-Nolte et al., 1990; 


$$
A(\omega, r)=S(\omega) C_{s}\left(\omega, r_{s}\right) R(\omega) C_{r}(\omega, r) G\left(\omega, r_{s}, r\right) \exp \left(-\frac{\omega}{2} Q_{p}^{-1} \Delta t_{r}\right)
$$

where $S$ and $R$ are the spectra of the source and the instrument response of the receiver, respectively; $r_{s}$ and $r$ are the depths of the source and the receiver, respectively; $\Delta t_{r}$ is the travel time of the $\mathrm{P}$-wave in the formation; and $Q_{p}^{-1}$ is an effective attenuation over the source-receiver offset $\left(r-r_{s}\right)$ that includes all intrinsic and extrinsic attenuation mechanisms except for geometrical spreading. The geometrical spreading $G$ is a function of frequency, depth, and source-receiver offset. The coupling terms of the source $C_{s}$ and of the receiver $C_{r}$ to the borehole are frequency-dependent. They include the attenuation of the P-wave during transmission through the fluid between the tool and the borehole wall.

Based on the expression given in Eq. 2, the effective attenuation $Q_{p}^{-1}$ at each frequency and for the travel path between two receivers can be computed as [e.g. Dasios et al., 2001; Baron and Holliger, 2010; Milani et al., 2015]

$$
Q_{p}^{-1}(\omega)=\ln \left(\frac{A\left(\omega, r_{i}\right) G_{i+1}}{A\left(\omega, r_{i+1}\right) G_{i}}\right) \frac{v_{p}(\omega)}{\pi f \Delta r}
$$

where $v_{p}$ is the $\mathrm{P}$-wave phase velocity in the formation between the $i$-th and $(i+1)$-th receivers, $\Delta r=\left|r_{i}-r_{i+1}\right|$, and $f=\omega / 2 \pi$. Eq. 3 is based on the assumptions that $R$ is the same for the two receivers and that the borehole wall is sufficiently uniform to consider $C_{r}$ as being independent of depth [Liang et al., 2017]. 
Eq. 3 implies that, in order to extract the effective attenuation $Q_{p}^{-1}$ from the recorded spectral amplitudes, we must correct for the losses associated with geometrical spreading. Moreover, in the case of an interval containing an individual fracture, we assume that the effective attenuation is a result of the intrinsic background attenuation and transmission losses across the fracture. The latter is the decrease in the transmitted P-wave amplitude caused by the energy conversion into reflected and transmitted waves at the fracture interfaces. The effective attenuation can therefore be quantified as

$$
Q_{p}^{-1}(\omega)=Q_{\text {raw }}^{-1}(\omega)-Q_{\text {sprd }}^{-1}(\omega)=Q_{0}^{-1}(\omega)+Q_{\text {transm }}^{-1}(\omega),
$$

where $Q_{r a w}^{-1}(\omega)=\ln \left(\frac{A\left(\omega, r_{i}\right)}{A\left(\omega, r_{i+1}\right)}\right) \frac{v_{p}(\omega)}{\pi f \Delta r}$ is the attenuation computed directly from the recorded amplitudes at two receivers, $Q_{s p r d}^{-1}(\omega)=\ln \left(\frac{G_{i}}{G_{i+1}}\right) \frac{v_{p}(\omega)}{\pi f \Delta r}$ is the attenuation due to geometrical spreading, $Q_{0}^{-1}(\omega)$ is the intrinsic attenuation of the background formation, and $Q_{\text {transm }}^{-1}(\omega)$ is the attenuation associated with transmission losses due to the presence of mesoscopic fractures, that is, fractures that are larger than the grain size but smaller than the prevailing sonic wavelengths. We are particularly interested in the last contribution to attenuation because it is related to the interaction of the sonic wave with the fractures and, hence, can be linked to their mechanical properties. In the following, we separate and remove the other contributions to the attenuation according to the relations given in Eq. 4 in order to estimate $Q_{\text {transm }}^{-1}$.

\subsubsection{Geometrical spreading correction}

One of the reasons for the decrease in amplitude of acoustic waves propagating along a borehole is geometrical spreading, which is represented in Eq. 3 by the symbols $G_{i}$ and $G_{i+1}$. Critically refracted compressional waves in boreholes are more complicated than analogous waves travelling along an interface between two half-spaces [Paillet and Cheng, 
1986]. Aki and Richards [2002] state that at sufficiently long offsets the amplitude decay of critically refracted waves travelling along a plane interface is proportional to $r^{-2}$, while a number of topical studies [e. g., Quan et al., 1994; Parra et al., 2007; Milani et al., 2015] have shown that the corresponding spreading characteristics along a borehole can be represented by a generic parametric function of the form

$$
G_{i}=\left(\frac{1}{r_{i}}\right)^{\gamma}
$$

where $\gamma$ is an empirical dimensionless parameter. This implies that the ratio of the spectral amplitudes of the signals recorded at two receivers located at distances $r_{i}$ and $r_{i+1}$ from the source in a homogeneous non-dissipative formation can be modelled as

$$
\frac{A\left(\omega, r_{i}\right)}{A\left(\omega, r_{i+1}\right)}=\left(\frac{r_{i+1}}{r_{i}}\right)^{\gamma}
$$

We explore two ways to estimate $\gamma$ and, consequently, the geometrical spreading correction. First, by performing numerical simulations and, second, from the FWS data using the overlap between short- and long-configuration measurements.

\subsubsection{Geometrical spreading correction estimated from synthetic data}

Following Milani et al. [2015], we perform numerical simulations of poroelastic seismic wave propagation in cylindrical coordinates based on Biot's (1962) dynamic equations for a rotationally symmetric medium [Sidler et al., 2013, 2014] to estimate the geometrical spreading correction factor $\gamma$ in Eq. 6. We assume an axisymmetric fluid-filled borehole surrounded by an isotropic porous formation. By doing so, we aim at modelling the geometrical spreading of the critically refracted P-wave travelling through the host rock under open borehole conditions. For this work, anisotropy effects on the modelling of the geometrical spreading characteristics are neglected. 
The considered borehole has a radius of $7.3 \mathrm{~cm}$, which corresponds to the nominal radius of the INJ2 borehole. We assume that the fluid saturating the borehole is water with a density $\rho_{f}$ of $1000 \mathrm{~kg} / \mathrm{m}^{3}$, a viscosity $\eta_{f}$ of 0.01 Poise, and a bulk modulus $K_{f}$ of $2.25 \mathrm{GPa}$. The physical properties of the formation are chosen based on ultrasonic $(f=1$ $\mathrm{MHz}$ ) velocity measurements reported in Wenning et al. [2018] made on dry core samples from a nearby borehole characterizing the granodiorite host rock. They measured P- and S-wave velocities and the sample's bulk density, porosity, and permeability. The shear and bulk moduli of the dry frame, $\mu$ and $K_{m}$, respectively, can be obtained using their relations with the $\mathrm{P}$ - and $\mathrm{S}$-wave velocities

$$
\begin{aligned}
\mu & =v_{s}^{2} \rho_{b}, \\
K_{m} & =v_{p}^{2} \rho_{b}-\frac{4 \mu}{3},
\end{aligned}
$$

where $\rho_{b}$ is the bulk density given by

$$
\rho_{b}=\rho_{f} \phi+\rho_{s}(1-\phi)
$$

with $\rho_{f}$ and $\rho_{s}$ being the fluid and grain densities, respectively, and $\phi$ the porosity. A strong foliation produces a pronounced velocity anisotropy, which Wenning et al. [2018] quantified by measuring velocities in two mutually orthogonal directions, one parallel and one perpendicular to the foliation (Table 2).

Notice that, in the less damaged zones of the borehole, the P-wave velocity computed from FWS logs lies between the laboratory estimates but is closer to that perpendicular to the foliation (Fig. 3). As we cannot account for the anisotropy of the rock in our numerical simulations, we consider the two sets of velocity measurements of Wenning et al. [2018] to compute the elastic moduli of the dry frame for the numerical simulations (Cases 1 and 2 in Table 3). The measured bulk density of the granodiorite is $2730 \mathrm{~kg} / \mathrm{m}^{3}$ 


\subsubsection{Geometrical spreading correction estimated from FWS data}

In the upper section of the borehole, where the two data sets of different source-receiver offsets were acquired (Table 1), the overlap in the position of the three receivers for both tool configurations allows us to estimate $\gamma$ directly from the FWS data by exploiting the redundancy of attenuation information in both measurements. Using the expression given in Eq. 4 and assuming a homogeneous formation over the length of the tool, the two different raw attenuation measurements can be approximated by

$$
\begin{aligned}
& Q_{\text {raw }, S}^{-1}(\omega)=Q_{p}^{-1}(\omega)+Q_{\text {sprd }, S}^{-1}(\omega), \\
& Q_{\text {raw }, L}^{-1}(\omega)=Q_{p}^{-1}(\omega)+Q_{\text {sprd }, L}^{-1}(\omega),
\end{aligned}
$$

where the subscripts $S$ and $L$ refer to the short and long configurations and $Q_{p}^{-1}$ is the effective attenuation in the interval between the receivers that is not due to geometrical spreading. When the surveyed intervals $\left[r_{S 1}-r_{S 2}\right]$ and $\left[r_{L 1}-r_{L 2}\right]$ coincide, we can assume that $Q_{p}^{-1}$ and $v_{p}(\omega)$ for the long and short configurations are the same. In this case, Eqs. 3 to 6 and 9 lead to

$$
\ln \left(\frac{A\left(\omega, r_{S 2}\right)}{A\left(\omega, r_{S 1}\right)}\right)+\gamma \ln \left(\frac{r_{S 2}}{r_{S 1}}\right)=\ln \left(\frac{A\left(\omega, r_{L 2}\right)}{A\left(\omega, r_{L 1}\right)}\right)+\gamma \ln \left(\frac{r_{L 2}}{r_{L 1}}\right)
$$

and we can compute $\gamma$ as

$$
\gamma=\frac{\left[-\ln \left(\frac{A\left(\omega, r_{S 2}\right)}{A\left(\omega, r_{S 1}\right)}\right)+\ln \left(\frac{A\left(\omega, r_{L 2}\right)}{A\left(\omega, r_{L 1}\right)}\right)\right]}{\left[\ln \left(\frac{r_{S 2}}{r_{S 1}}\right)-\ln \left(\frac{r_{L 2}}{r_{L 1}}\right)\right]} .
$$


404

It is important to mention that we have verified the validity of Eq. 11 by performing numerical simulations in a homogeneous borehole. In the following, we only apply this procedure to the real data. Fig. 5a shows $\gamma$ computed from Eq. 11 as a function of depth (dots) in the upper section. For robustness, for each depth position, we compute the mean $\gamma$ from the values obtained for nominal source frequencies of 15 and $25 \mathrm{kHz}$. We observe that $\gamma$ is larger in the damaged zones, that is, in the presence of fractures. In these zones, Eq. 5, which assumes homogeneity, and, hence, the methodology given by Eqs. 9 to 11, are not valid to describe the geometrical spreading. Correspondingly, the obtained values are not strictly comparable with those inferred from the numerical simulations as the latter assume a homogeneous formation. Conversely, in the intervals where the formation is less damaged, $\gamma$ is smaller and approaches the range of values obtained from numerical simulations (blue dashed lines).

In the lower section of the borehole, we do not have a combination of long- and shortconfiguration measurements, but the shorter distance between consecutive source locations, results in an overlap in the receivers positions for different pairs of offsets to the source. That is, as the tool moves upwards along the borehole, the interval surveyed by $\mathrm{Rx} 2$ and $\mathrm{Rx} 3$ for the $i$-th source location will be surveyed also by $\mathrm{Rx} 1$ and $\mathrm{Rx} 2$ for the $(i+1)$-th source location but with different source-receiver offsets. Using this, we can estimate $\gamma$ in the same way as for the upper section. Fig. 5b shows a mean value of ${ }_{23} \gamma$ computed using the data for nominal source frequencies of 15 and $25 \mathrm{kHz}$ (dots). We observe that, as before, $\gamma$ increases in the vicinity of fracture zones and decreases to values similar to those predicted by the numerical model in the less damaged zones. 
${ }_{443} \mathrm{Rx} 2$ and $\mathrm{Rx} 3$.

444 
${ }_{449}$ Small discrepancies are indeed expected due to the effects of interfering wave modes in the spectrum of the two-cycle wavelet. From this comparison, we conclude that the inferred

${ }_{451}$ attenuation profiles are robust with respect to the isolation of the critically refracted ${ }_{452} \mathrm{P}$-wave.

${ }_{453}$ In general, Figs. 6 to 8 show that the depth dependence of the attenuation is similar ${ }_{454}$ for both frequencies and that attenuation slightly decreases with frequency. Although $\gamma$ ${ }_{455}$ is relatively low, the geometrical spreading represents a significant contribution to the ${ }_{456}$ overall attenuation. Given that the nominal source frequencies are very close to each ${ }_{457}$ other, we use a constant value of 0.5 for $\gamma$.

From the televiewer images, the velocity and $\gamma$ profiles, we can identify zones with in-

${ }_{460} Q_{0}^{-1}$ (Eq. 4) is independent of depth in each of the analyzed sections, we can estimate it

${ }_{461}$ by defining a mean value for the corrected attenuation in the less damaged zones. Figs.

${ }_{462} 6$ to 8 show that this attenuation baseline lies between 0.069 and 0.082 , corresponding to

${ }_{463} Q_{0}$-values between 12 and 14.5, depending on the depth. We observe that the intrinsic

${ }_{464}$ background attenuation tends to decrease with depth. Given that the degree of inelastic-

${ }_{465}$ ity depends on the composition of the rock (matrix minerals, porosity, pore fluids) and

${ }_{466}$ the in situ pressure and temperature [Dasios et al., 2001], one possible explanation for the

${ }_{467}$ lower attenuation values in the lower section of the borehole may be the differences be-

${ }_{468}$ tween the properties of the ductile shear zone (green shadow zone) and the less deformed

${ }_{469}$ granodioritic host rock.

470 Lastly, it is important to mention that the high attenuation values resulting from our ${ }_{471}$ analysis are in agreement with previously reported estimates. Cosma and Enescu [2001] 
${ }_{472}$ suggested that, due to heavy deformation during the Alpine orogeny, relatively high values ${ }_{473}$ for $Q_{p}^{-1}$ of 0.05 to 0.1 are to be expected for the Palaeozoic granodiorite at the GTS. ${ }_{474}$ Majer et al. [1990] performed a tomographic analysis of crosshole data from multiple ${ }_{475}$ offsets and azimuths at the GTS and estimated a $Q_{0}^{-1}$ value of 0.083 for the background 476 rock at $6 \mathrm{kHz}$. Holliger and Bühnemann [1996] reported $Q_{p}^{-1}$ values acquired at the ${ }_{477}$ GTS using high-quality seismic data in a frequency range between 50 and $1500 \mathrm{~Hz}$. The ${ }_{478}$ corresponding estimates lie between 0.016 and 0.05 with a median value of 0.029 , which 479 are again consistent with our estimates.

\section{Effect of individual fractures on the attenuation and phase velocity of sonic} waves

${ }_{480}$ In the previous section, we have shown that the geometrical spreading and inelasticity ${ }_{481}$ in the background can have a significant impact on the observed attenuation between ${ }_{482}$ two receivers. However, Figs. 6 to 8 show that attenuation also increases in zones with ${ }_{483}$ fractures or dykes with respect to the background attenuation. In this case, the observed ${ }_{484}$ increase of attenuation is expected to be related to transmission losses across these hetero${ }_{485}$ geneities. This is the case, for example, for the extremely high attenuation value observed ${ }_{486}$ at $25 \mathrm{~m}$ depth in Fig. 7, which is associated with the presence of a fractured lamprophyre ${ }_{487}$ dyke (Fig. 9a). On the other hand, the peak attenuation observed at a depth of $\sim 8 \mathrm{~m}$ is ${ }_{488}$ related to the presence of a fracture (Fig. 9b). Based on the results of the previous section,

${ }_{489}$ we can isolate the attenuation due to transmission losses by removing the effects due to 490 geometrical spreading and intrinsic background attenuation (Eq. 4). In this section, we ${ }_{491}$ use the transmission losses due to the presence of fractures as well as the corresponding ${ }_{492}$ phase velocity changes to infer the fracture mechanical normal compliance. 


\subsection{Transmission losses and fracture compliance}

499 et al., 2009]

$$
T(\omega)=\frac{1}{1+\frac{i \omega I_{b} Z_{N}}{2}},
$$

with $T$ denoting the $\mathrm{P}$-wave transmission coefficient at normal incidence and $I=\rho v_{p}$ the impedance. The subscript $b$ refers to background rock properties. Given that Eq. 12 is strictly valid for normal incidence, $Z_{N}$ corresponds to the so-called normal compliance of the fracture. The effective compliance of the fracture can then be estimated from the transmission coefficient as

$$
Z_{N}=\frac{(1-T)}{i T} \frac{2}{\omega I_{b}} .
$$

Note that Eq. 12 corresponds to the transmission coefficient associated with an interface that represents a plane of weakness in the rock [Schoenberg, 1980]. In the limit of $Z_{N} \rightarrow 0$, the case of a welded interface is approached and $T \rightarrow 1$. In the following, we therefore focus on fractures that are more compliant than the embedding background, which are identified by a decrease in the P-wave velocity (Fig. 3). Moreover, Eq. 13 allows the compliance to be complex-valued [Schoenberg, 1980]. The imaginary and real components of the compliance can be used not only to determine the weakening effect of the fracture on the rock but also to get information about possible mechanisms of energy dissipation occurring 
In order to estimate the complex-valued mechanical compliance of the fractures from Eq. 13, we must first obtain the P-wave transmission coefficient. Given that Eq. 13 was derived to model the effect of a fracture on the propagation of plane seismic waves [Pyrak-Nolte et al., 1990], we perform numerical simulations to demonstrate that the attenuation corrected for geometrical spreading and the phase velocity computed from sonic logs are similar to those obtained for a plane-wave propagating through a medium containing a planar fracture of infinite horizontal extent (Appendix A). As a consequence of their similarity, the complex-valued $\mathrm{P}$-wave transmission coefficient $T$ associated with the presence of a fracture can be computed as

$$
T=e^{i\left(k_{p}^{b}-k_{p}^{e f f}\right) \Delta r}
$$

where $k_{p}^{b}$ and $k_{p}^{e f f}$ correspond to the wavenumber of the background rock and the wavenumber of an effective viscoelastic medium representing the fractured section between two receivers, respectively, and $\Delta r$ is the separation between the receivers. Both wavenumbers can be obtained from the velocity and attenuation computed from the FWS data as

$$
k_{p}=\frac{\omega}{v_{p}}\left[1-i \frac{Q_{p}^{-1}}{2}\right]
$$

where we have approximated the attenuation as $Q_{p}^{-1} \approx-2 \frac{\Im\left[k_{p}\right]}{\Re\left[k_{p}\right]}[$ Pride, 2005]. 
544 results for both nominal frequencies as well as applying or not applying the geometrical

${ }_{545}$ spreading correction to the attenuation values when computing the wavenumbers in Eq.

${ }_{546}$ 15. In Table 4, we therefore simply present an average of all those estimates.

The real component of the mechanical compliance of the analyzed fractures was found ${ }_{548}$ to lie in the range between $\sim 1 \times 10^{-13} \mathrm{~m} / \mathrm{Pa}$ and $\sim 1 \times 10^{-12} \mathrm{~m} / \mathrm{Pa}$. Figs. $9 \mathrm{~b}$ and 10 show that the fractures intersect the borehole at different angles. In Table 4, we approximate 
to normal incidence. For incidence angles lower than $60^{\circ}$, both the real and imaginary components of the compliance are not particularly sensitive to the incidence angle at which the transmission coefficient was computed. However, for larger incidence angles, which correspond to the case of steeply dipping fractures with respect to the borehole trajectory, the imaginary component of the estimated compliance becomes comparable to the real component and both are less representative of the correct normal compliance. From this analysis, we expect that the overestimation of the compliances may be more important for the fractures at $\sim 21.8$ and $\sim 23.1 \mathrm{~m}$.

Furthermore, notice that the imaginary components of the estimated fracture compliances are not negligible (Table 4). As discussed above, one possible reason for the relatively high imaginary component of the compliance is due to steep dips $\theta_{D}$. However, we observe a large imaginary component for all of the fractures and not only for those with associated large value of $\theta_{D}$. Hence, the importance of the imaginary component of the compliance is more likely to be related to damping effects occurring in the fracture. One possible damping mechanism is WIFF between the fracture and the background. Due to the very low permeability of the background rock of the order of tens of $\mu$ Darcy, the characteristic frequency, at which WIFF effects arise, is expected to be significantly below the nominal frequencies of the FWS logs. Therefore, the contribution of mesoscopic WIFF should be negligible. However, these effects cannot be completely ruled out as, for example, the presence of microcracks in the vicinity of the fractures can effectively increase the permeability of the rock surrounding the fracture. This in turn, may enhance the effects due to mesoscopic WIFF and shift their characteristic frequency towards the 
${ }_{589}$ sonic range as well as produce additional energy dissipation due to squirt-flow effects at

the microscale [e.g. Müller et al., 2010].

Regarding the relative variation of the compliance estimates for different sections of the borehole, we found that fractures exhibit compliance values that are almost an orderof-magnitude larger in the central section than in the other sections. Fig. 12 shows a zero-offset hydrophone vertical seismic profile (VSP) section composed of traces registered at depths ranging from 11.5 to $44 \mathrm{~m}$ depth along the INJ2 borehole. When an external wave field is incident on a fluid-filled open fracture intersecting a borehole, it squeezes the fracture and expels fluid into the borehole thus generating a so-called tube wave [Bakku et al., 2013]. We have found two typical chevron-type patterns associated with the propagation of tube waves (red dashed lines). These two strong tube wave signatures intersect the borehole at $\sim 23.5$ and $\sim 25$ m depth, which coincide with fractures observed in the televiewer images. We have not computed the compliance for the fracture located at $\sim 25 \mathrm{~m}$ depth as the velocity and attenuation are strongly affected by the presence of a lamprophyre dyke (Fig. 9a). However, the fact that the highest estimated compliance inferred for the fracture intersecting the borehole at $\sim 23.5 \mathrm{~m}$ depth (Table 4 ) coincides with strong tube wave generation points to the sensitivity of the estimations to the implicit relation between fracture compliance and its hydraulic transmissivity [e.g. Pyrak-Nolte and Morris, 2000]. In this regard, heat dilution tests performed by Jalali et al. [2018] in the injection boreholes of GTS revealed a zone of enhanced cooling at $23.5 \mathrm{~m}$ borehole depth in the INJ2 indicating the presence of hydraulically highly conductive fractures.

\subsubsection{Comparison with literature values}


It is interesting to compare the estimated fracture compliances with those previously reported in the literature. Fig. 13 shows fracture compliances compiled from laboratory and seismic field experiments by Worthington and Lubbe [2007] and in Table 1 of Hobday and Worthington [2012] and references therein. The blue and red colours indicate laboratory and field measurements, respectively, after Zangerl et al. [2008]. For completeness, we also include in Fig. 13 the compliance estimates reported by Baird et al. [2013], Bakku et al. [2013], Verdon and Wüstefeld [2013], Nakagawa [2013], and Minato et al. [2017] after the publication of Hobday and Worthington [2012]. The estimations of Bakku et al. [2013] for meter-scale fractures, which are represented with a dotted line, were computed using tube wave amplitudes and correspond to the same fractures studied by Hardin et al. [1987] (red solid line at $1 \mathrm{~m}$ fracture size). However, Hardin et al. [1987] considered a lowfrequency approximation for the flow in the fractures, which leads to an underestimation of the compliance. Nevertheless, it is insightful to note the range of variability that fracture compliances can assume depending on the model used. Moreover, we have computed the effective compliances of the cracks composing the synthetic sample of Rathore et al. [1995] by using their velocity anisotropy measurements after Barbosa et al. [2018] (green dot in Fig. 13).

The real and absolute values of the compliance estimates obtained in this work (Table 4) are indicated in Fig. 13 with black and grey ellipses, which, in turn, reflect the uncertainties with regard to the sizes of the fractures. Gischig et al. [2018] carried out hydrofracturing tests in a nearby borehole in GTS as part of a stress characterization survey. The resulting seismicity clouds have diameters of the order of $5 \mathrm{~m}$. Jalali et al. [2018] performed a series of geophysical and hydrological tests on the injection boreholes of GTS for 


\section{Discussion and conclusions}


${ }_{713}$ elastic properties of the fractured rock.

714

associated to changes on the shape, compliance, and orientation of contact areas along the fracture that produce compressibility contrasts at the microscale of the fracture. Another explanation for the viscoelastic behavior of fractures to the transmission of seismic waves has been proposed by Yoshioka and Kikuchi [1993] for ultrasonic frequencies. In that case, the authors argued that the deviation of the response of a fracture from purely elastic can be associated to plastic behavior at the asperities of the fracture caused by high local pressure. However, as the imaginary component of the fracture compliance is generally smaller than its real counterpart, it is also expected to be more affected by uncertainties in the attenuation and phase velocity estimations as well as by the dipping angle of the fracture (Fig. 11). Further investigation needs to be done in order to elucidate the origin of the complex nature of the fracture compliance and its relation to the hydraulic and Acknowledgments. This work was supported by a grant from the Swiss National Science Foundation and completed within SCCER-SOE with the support of Innosuisse. We thank Quinn Wenning and Claudio Madonna for providing laboratory measurements as well as Joseph Doetsch and Hannes Krietsch for the televiewer images and the interpretation thereof. Tobias Zahner made essential contributions with regard to planning and execution of the field experiments. J. Germán Rubino gratefully acknowledges an extended visit to the University of Lausanne financed by the Fondation Herbette. The authors gratefully acknowledge comments and suggestions from three anonymous referees and from the editor Douglas Schmitt. The data associated with this paper are available from https://github.com/rockphysicsUNIL/GTS_FWS_data. 


\section{Appendix A: Methodology to estimate the complex-valued transmission} coefficient from attenuation and velocity measurements 
absence of heterogeneity, the estimated velocity is, as expected, close to the background velocity (black line) and the attenuation is negligible. Notice that the velocities computed from numerical simulations for the intact rock model $\left(v_{p} \sim 5150 \mathrm{~m} / \mathrm{s}\right)$ underestimate the velocity of the background $\left(v_{p}=5220 \mathrm{~m} / \mathrm{s}\right)$. However, the maximum relative difference between them is $\sim 1.3 \%$, which is small and similar to uncertainties commonly associated with phase velocity estimations [Moos and Zoback, 1983; Molyneux and Schmitt, 2000; McCann and Sothcott, 2009].

In the presence of a compliant layer between receivers, the effective velocity measured is lower than the background velocity and gets closer to the latter as the distance between receivers increases. The attenuation shows low values when both receivers are located before the layer. Some attenuation values are negative, which may be due to strong scattering effects close to the thin layer and, to a lesser degree, to an incorrect geometrical spreading correction. As the distance between receivers increases, the attenuation describes a more predictable and decreasing behavior. The reason for the decrease in attenuation is that the transmission losses remain the same but the total distance covered by the $\mathrm{P}$-wave is larger and, hence, the effective attenuation is lower.

The numerical results show a significant impact of the presence of thin layers on both the attenuation and velocity estimates. Hence, they suggest that it may be possible to extract information about the thin layer properties from transmission losses and effective velocities. In the following, we will show that the attenuation and velocity behavior depicted in Fig. 14 can be modelled with the solution of a plane-wave propagating in a fluid-saturated poroelastic medium containing a single porous layer. We refer to this 
model as the thin-layer model. For details regarding this plane-wave solution, we refer the reader to Barbosa et al. [2016].

The thin-layer model allows us to compute any poroelastic field in frequency-space domain resulting from the contributions of all the wave modes generated from the incidence of a seismic wave on a thin layer. For a normally incident P-wave, the incident $\left(u^{i}\right)$ and transmitted $\left(u^{t}\right)$ solid displacement fields are given by

$$
\begin{aligned}
& u_{y_{1}}^{i}=-i k_{p} \exp \left[-i k_{p}\left(-y_{1}\right)\right], \\
& u_{y_{2}}^{t}=-i k_{p} T \exp \left[-i k_{p}\left(y_{2}\right)\right],
\end{aligned}
$$

where $y_{1}>0$ and $y_{2}>0$ are the offsets of receivers 1 and 2 , respectively, from the upper interface of the layer $(y=0)$. We assume that receivers 1 and 2 are located before and after the layer, respectively. $T$ is the $\mathrm{P}$-wave transmission coefficient and $k_{p}$ is the $\mathrm{P}$-wave number in the background medium (Eq. 15). The sign of the real part of $k_{p}$ is positive for waves traveling in the direction of increasing $y$ as in Barbosa et al. [2016]. By using Eq. 16 we exclude the displacements associated with the slow P-wave as well as the reflections from the layer, assuming that only the incident and transmitted fields contribute to the signals recorded at the two receivers.

In order to obtain the effective attenuation, we assume that the decay in the P-wave solid displacement fields in the interval between $y_{1}$ and $y_{2}$ can be explained by a homogeneous viscoelastic medium. By doing so, we can obtain an effective P-wave number as a function of the background properties and the transmission coefficient $T$

$$
k_{p}^{e f f}=\frac{-i k_{p} d y+\ln [T]}{-i d y} .
$$


${ }_{790}$ Notice that the solution of the plane-wave propagation across a single layer does not

${ }_{791}$ only account for the scattering effects but also for the WIFF effects resulting from the ${ }_{792}$ poroelastic representation of the model.

$$
\begin{aligned}
Q_{p}^{-1} & =-\frac{\Im\left[\left(k_{p}^{e f f}\right)^{2}\right]}{\Re\left[\left(k_{p}^{e f f}\right)^{2}\right]}, \\
v_{p} & =\frac{\omega}{\Re\left[k_{p}^{e f f}\right]} .
\end{aligned}
$$

Fig. 14 shows the velocity and attenuation for the thin-layer model (solid blue curves) computed using Eqs. 17 and 18 and the transmission coefficient obtained from the planewave analysis performed by Barbosa et al. [2016]. Although the results for the thin-layer model only depend on the distance between the receivers located before and after the layer $(d y)$ we assume, for illustration purposes, that $y_{1}=0.35 \mathrm{~m}$ (before the layer) and $y_{2}$ ranges from 0.05 to $0.45 \mathrm{~m}$ (after the layer). We observe that the overall agreement between the attenuation and velocity from the numerical simulations and the thin-layer model is very good at relatively large offsets between receivers where the influence of the scattered waves from the layer on the critically refracted P-wave decreases and the numerical results stabilize. It can be shown that the interference between the direct critically refracted Pwave and that reflected at the fracture is negligible for a distance between the receiver and the fracture larger than $T * v_{p} / 2$, where $\mathrm{T}$ is the wave period. Lastly, it is important to remark that we have used the geometrical spreading coefficient of the intact background to correct the attenuation estimates from the borehole code. These results imply that at large distances between receivers, the impact of the fracture properties on the geometrical spreading correction is negligible. 


\section{Appendix B: Validation of the methodology to estimate the complex-valued} transmission coefficient from attenuation and velocity measurements 
${ }_{837}$ them.

838

846 values.

\section{References}


behavior during deep geothermal reservoir stimulations: open questions tackled in a decameterscale in situ stimulation experiment, Solid Earth, 9, 115-137.

Baird, A. F., J. M. Kendall, and D. A. Angus (2013), Frequency-dependent seismic anisotropy due to fractures: Fluid flow versus scattering, Geophysics, 78, WA111WA122.

Bakku, S. K., M. Fehler, and D. Burns (2013), Fracture compliance estimation using borehole tube waves, Geophysics, 78, 249-260.

Barbosa, N. D., J. G. Rubino, E. Caspari, M. Milani, and K. Holliger (2016), Fluid pressure diffusion effects on the seismic reflectivity of a single fracture, The Journal of the Acoustical Society of America, 140, 2554-2570.

Barbosa, N. D., J. G. Rubino, E. Caspari, and K. Holliger (2017), Extension of the classical linear slip model for fluid-saturated fractures: Accounting for fluid pressure diffusion effects, Journal of Geophysical Research: Solid Earth, 122, 1302-1323.

Barbosa, N. D., J. G. Rubino, E. Caspari, and K. Holliger (2018), Impact of fracture clustering on the seismic signatures of porous rocks containing aligned fractures, Geophysics, 83, 295-308.

Baron, L., and K. Holliger (2010), Analysis of the velocity dispersion and attenuation behavior of multifrequency sonic log, Advances in Near-Surface Seismology and GroundPenetrating Radar, 153-166.

Biot, M. A. (1956), Theory of propagation of elastic waves in a fluid-saturated porous solid. I. Low-frequency range, The Journal of the acoustical Society of america, 28, 168-178. 
Biot, M. A. (1956), Mechanics of deformation and acoustic propagation in porous media, Journal of Applied Physics, 33, 1482-1498.

Chapman, M. (2003), Frequency-dependent anisotropy due to meso-scale fractures in the presence of equant porosity, Geophysical Prospecting, 51, 369-379.

Cheng, C. H., M. N. Toksöz, and M. E. Willis (1982), Determination of in situ attenuation from full waveform acoustic logs, Journal of Geophysical Research: Solid Earth, 87, $5477-5484$

Cosma, C., and N. Enescu (2001), Characterization of fractured rock in the vicinity of tunnels by the swept impact seismic technique, International Journal of Rock Mechanics and Mining Sciences, 38, 815-821.

Dasios, A., T. R. Astin, and C. McCann (2001), Compressional-wave Q estimation from full-waveform sonic data, Geophysical Prospecting, 49, 353-373.

Delay, J., P. Bossart, L. X. Ling, I. Blechschmidt, M. Ohlsson, A. Vinsot, C. Nussbaum, and N. Maes (2014), Three decades of underground research laboratories: what have we learned?, Geological Society, London, Special Publications, 400, 7-32.

Fang, X., Y. Zheng, and M. C. Fehler (2017), Fracture clustering effect on amplitude variation with offset and azimuth analyses, Geophysics, 82, N13-N25.

Faulkner, D. R., T. M. Mitchell, E. Jensen, and J. Cembrano (2011), Scaling of fault damage zones with displacement and the implications for fault growth processes, Journal of Geophysical Research: Solid Earth, 116.

Gischig, V. S., J. Doetsch, H. Maurer, H. Krietsch, F. Amann, K. F. Evans, and S. Wiemer (2018), On the link between stress field and small-scale hydraulic fracture growth in anisotropic rock derived from microseismicity, Solid Earth, 9, 39. 
Gu, B., R. Suárez-Rivera, K. T. Nihei, and L. R. Myer (1996), Incidence of plane waves upon a fracture, Journal of Geophysical Research: Solid Earth, 101, 25337-25346.

Guerin, G., and D. Goldberg (2002), Sonic waveform attenuation in gas hydrate-bearing sediments from the Mallik 2L-38 research well, Mackenzie Delta, Canada, Journal of Geophysical Research: Solid Earth, $10 \%$.

Gurevich, B. (2003), Elastic properties of saturated porous rocks with aligned fractures, Journal of Applied Geophysics, 54, 203-218.

Hardin, E. L., C. H. Cheng, F. L. Paillet, and J. D. Mendelson (1987), Fracture characterization by means of attenuation and generation of tube waves in fractured crystalline rock at Mirror Lake, New Hampshire, Journal of Geophysical Research: Solid Earth, 92, 7989-8006.

Hobday, C., and M. H. Worthington (2012), Field measurements of normal and shear fracture compliance, Geophysical Prospecting, 60, 488-499.

Holliger, K., and J. Bühnemann (1996), Attenuation of broad-band (50-1500 Hz) seismic waves in granitic rocks near the Earth's surface, Geophysical Research Letters, 23, 19811984 .

Hudson, J. A. (1980), Overall properties of a cracked solid, Mathematical Proceedings of the Cambridge Philosophical Society: Cambridge University Press, 88, 371-384.

Hudson, J. A., E. Liu, and S. Crampin (1996), Transmission properties of a plane fault, Geophysical Journal International, 125, 559-566.

Jaeger, J. C., N. G. Cook, and R. Zimmerman (2009), Fundamentals of rock mechanics. John Wiley and Sons. 
Jalali, M. R., V. Gischig, J. Doetsch, H. Krietsch, F. Amann, and M. Klepikova (2017), Mechanical, Hydraulic and Seismological Behavior of Crystalline Rock as a Response to Hydraulic Fracturing at the Grimsel Test Site, In 51st US Rock Mechanics/Geomechanics Symposium, American Rock Mechanics Association.

Jalali, M. R., M. Klepikova, J. Doetsch, H. Krietsch, B. Brixel, N. Dutler, V. Gischig, and F. Amann (2018), A Multi-Scale Approach to Identify and Characterize Preferential Flow Paths in a Fractured Crystalline Rock, In 2nd International Discrete Fracture Network Engineering Conference, American Rock Mechanics Association.

Jalali, M., V. Gischig, J. Doetsch, R. Näf, H. Krietsch, M. Klepikova, F. Amann, and D. Giardini (2018), Transmissivity Changes and Microseismicity Induced by SmallScale Hydraulic Fracturing Tests in Crystalline Rock, Geophysical Research Letters, 45, 22652273.

Kang, P. K., Y. Zheng, X. Fang, R. Wojcik, D. McLaughlin, S. Brown, M. C. Fehler, D. R. Burns, and R. Juanes (2016), Sequential approach to joint flowseismic inversion for improved characterization of fractured media, Water Resources Research, 52, 903-919.

Keusen, H. R., J. Ganguin, P. Schuler, and M. Buletti (1989), Grimsel Test Site-Geology. (Rep. NTB 87-14E). Baden: Nagra.

Krietsch, H., V. Gischig, M. R. Jalali, F. Amann, K. F. Evans, J. Doetsch, and B. Valley (2017), Stress measurements in crystalline rock: Comparison of overcoring, hydraulic fracturing and induced seismicity results, In 51st US Rock Mechanics/Geomechanics Symposium, American Rock Mechanics Association.

Krietsch, H., J. Doetsch, N. Dutler, M. Jalali, V. Gischig, S. Loew, and F. Amann (2018), Comprehensive geological dataset for a fractured crystalline rock volume at the Grimsel 
Test Site. DOI:10.3929/ethz-b-000243199.

Liang, J., T. M. Müller, G. Tang, and Q. Qi (2017), Towards developing a robust Qextraction method from full-waveform acoustic logs, In 79th EAGE Conference and Exhibition 201\%.

Liu, E., J. A. Hudson, and T. Pointer (2000), Equivalent medium representation of fractured rock, Journal of Geophysical Research: Solid Earth, 105, 2981-3000.

Lubbe, R., and M. Worthington (2006), A field investigation of fracture compliance, Geophysical Prospecting, 54, 319-331.

Lubbe, R., J. Sothcott, M. H. Worthington, and C. McCann (2008), Laboratory estimates of normal and shear fracture compliance, Geophysical Prospecting, 56, 239-247.

Majer, E. L., L. R. Myer, J. E. Peterson Jr, K. Karasaki, J.C. S. Long, S. J. Martel, P. Blümling, and S. Vomvoris (1990), Joint seismic, hydrogeological, and geomechanical investigations of a fracture zone in the Grimsel Rock Laboratory, Switzerland. (No. LBL-27913; NDC-14). Lawrence Berkeley Lab., CA (USA); Nationale Genossenschaft fuer die Lagerung Radioaktiver Abfaelle (NAGRA), Baden (Switzerland).

McCann, C., and J. Sothcott (2009), Sonic to ultrasonic Q of sandstones and limestones: Laboratory measurements at in situ pressures, Geophysics, 74, 93-101.

Milani, M., J. G. Rubino, L. Baron, R. Sidler, and K. Holliger (2015), Attenuation of sonic waves in water-saturated alluvial sediments due to wave-induced fluid flow at microscopic, mesoscopic and macroscopic scales, Geophysical Journal International, 203, $146-157$.

Minato, S., and R. Ghose (2016), AVO inversion for a non-welded interface: estimating compliances of a fluid-filled fracture, Geophysical Journal International, 206, 56-62. 
${ }_{964}$ Minato, S., R. Ghose, and G. Osukuku (2017), Experimental verification of spatially varying fracture-compliance estimates obtained from AVO inversion coupled with linear slip theory, Geophysics, 83, 1-38.

Möllhoff, M., C. J. Bean, and P. G. Meredith (2010), Rock fracture compliance derived from time delays of elastic waves, Geophysical Prospecting, 58, 1111-1122.

Molyneux, J. B., and D. R. Schmitt (2000), Compressional-wave velocities in attenuating media: A laboratory physical model study, Geophysics, 65, 1162-1167.

Moos, D., and M. D. Zoback (1983), In situ studies of velocity in fractured crystalline rocks, Journal of Geophysical Research: Solid Earth, 88, 2345-2358.

Morris, R. L., D. R. Grine, and T. E. Arkfeld (1964), Using compressional and shear acoustic amplitudes for the location of fractures, Journal of Petroleum Technology, 16, 623-632.

Müller, T. M., B. Gurevich, and M. Lebedev (2010), Seismic wave attenuation and dispersion resulting from wave-induced flow in porous rocks: A review, Geophysics, 75, $147-164$.

Nakagawa, S. (2013), Low-frequency $(<100 \mathrm{~Hz})$ dynamic fracture compliance measurement in the laboratory, ARMA-2013-343. In 47th US Rock Mechanics/Geomechanics Symposium, 23-26 June, San Francisco, Am. Rock Mech. Assoc.

Paillet, F. L., and C. H. Cheng (1986), A numerical investigation of head waves and leaky modes in fluid-filled boreholes, Geophysics, 51, 1438-1449.

Parra, J. O., P. C. Xu, and C. L. Hackert (2007), A borehole-model-derived algorithm for estimating $Q_{P}$ logs from full-waveform sonic logs, Geophysics, 72, 107-117. 
Pyrak-Nolte, L. J., and D. D. Nolte (1992), Frequency dependence of fracture stiffness, Geophysical Research Letters, 19, 325-328.

Pyrak-Nolte, L. J., L. R. Myer, and N. G. W. Cook (1990), Transmission of seismic waves across single natural fractures, J. Geophys. Res., 95, 8617-8638.

Pyrak-Nolte, L. J., and J. P. Morris (2000), Single fractures under normal stress: The relation between fracture specific stiffness and fluid flow, International Journal of Rock Mechanics and Mining Sciences, 37, 245-262.

Pyrak-Nolte, L. J., and D. D. Nolte (2016), Approaching a universal scaling relationship between fracture stiffness and fluid flow, Nature communications, 7, 10663.

Pride, S. R. (2005), Relationships between seismic and hydrological properties, in Hydrogeophysics, pp. 253-290. Springer Netherlands.

Prioul, R., and J. Jocker (2009), Fracture characterization at multiple scales using borehole images, sonic logs, and walkaround vertical seismic profile, AAPG bulletin, 93, 15031516.

Qi, Q., T. M. Müller, and M. Pervukhina (2017), Sonic $Q_{P} / Q_{S}$ ratio as diagnostic tool for shale gas saturation, Geophysics, 82, 97-103.

Quan, Y., J. M. Harris, and X. Chen (1994), Acoustic attenuation logging using centroid frequency shift and amplitude ratio methods: A numerical study, In SEG Technical Program Expanded Abstracts 1994, Society of Exploration Geophysicists.

Rathore, J. S., E. Fjaer, R. M. Holt, and L. Renlie (1995), P-and S-wave anisotropy of a synthetic sandstone with controlled crack geometry, Geophysical Prospecting, 43, $711-728$. 
Rubino, J. G., L. Guarracino, T. M. Müller, and K. Holliger (2013), Do seismic waves sense fracture connectivity?, Geophysical Research Letters, 40, 692-696.

Rutqvist, J. (2015), Fractured rock stress-permeability relationships from in situ data and effects of temperature and chemical-mechanical couplings, Geofluids, 15, 48-66.

Sams, M. (1991), High-resolution velocity and attenuation logs from long-spaced sonic data, Geophysics, 56, 1071-1080.

Schoenberg, M. A. (1980), Elastic wave behavior across linear slip interfaces, J. Acoust. Soc. Am., 68, 1516-1521.

Schoenberg, M., and J. Douma (1988), Elastic wave propagation in media with parallel fractures and aligned cracks, Geophysical Prospecting, 36, 571-590.

Sidler, R., J. M. Carcione, and K. Holliger (2013), A pseudo-spectral method for the simulation of poro-elastic seismic wave propagation in 2D polar coordinates using domain decomposition, Journal of Computational Physics, 235, 846-864.

Sidler, R., J. M. Carcione, and K. Holliger (2014), A pseudospectral method for the simulation of 3-D ultrasonic and seismic waves in heterogeneous poroelastic borehole environments, Geophysical Journal International, 192, 1134-1151.

Sil, S. (2013), Fracture parameter estimation from well-log data, Geophysics, 78, D129D134.

Sun, X., X. Tang, C. H. Cheng, and L. N. Frazer (2000), P-and S-wave attenuation logs from monopole sonic data, Geophysics, 65, 755-765.

Verdon, J. P., and A. Wüstefeld (2013), Measurement of the normal/tangential fracture compliance ratio (ZN/ZT) during hydraulic fracture stimulation using S-wave splitting data, Geophysical Prospecting, 61, 461-475. 
$\mathrm{X}-50$

Wenning, Q., C. Madonna, A. de Haller, and J. P. Burg (2018), Permeability and seismic velocity anisotropy across a ductile-brittle fault zone in crystalline rock, Solid Earth, 9, 683-698.

Worthington, M. H., and J. A. Hudson (2000), Fault properties from seismic Q, Geophysical Journal International, 143, 937-944.

Worthington, M. H., and R. Lubbe (2007), The scaling of fracture compliance, Geological Society (London) Special Publications, 270, 73-82.

Yoshioka, N., and M. Kikuchi (1993), Visco-elastic response of joints to transmission waves, Geophysical research letters, 20, 1143-1146.

Zangerl, C., E. Eberhardt, K. Evans, and S. Löw (2008), Normal stiffness of fractures in granitic rock: a compilation of laboratory and in-situ experiments, International journal of rock mechanics and mining sciences, 45, 1500-1507.

Zimmerman, R., and I. Main (2004), Hydromechanical behavior of fractured rocks, International Geophysics Series, 89, 363-422. 


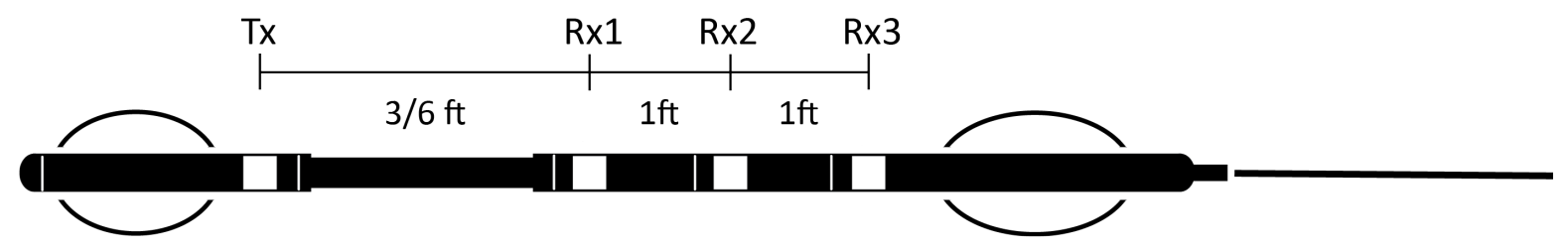

Figure 1. Schematic illustration of the used sonic logging tool with one transmitter (Tx) and three receivers $(\mathrm{Rx} 1, \mathrm{Rx} 2, \mathrm{Rx} 3)$. The offset to the source of the first receiver is 3 and $6 \mathrm{ft}$ for the short and long tool configurations, respectively.

Table 1. Transmitter positions along the borehole. SC and LC refer to short and long tool configurations, respectively.

SC depth range LC depth range Spatial sampling rate

\begin{tabular}{lccc}
\hline Upper section & $4.89-9.69[\mathrm{~m}]$ & $7.60-10.60[\mathrm{~m}]$ & $0.60[\mathrm{~m}]$ \\
Central section & $19.49-26.69[\mathrm{~m}]$ & - & $0.60[\mathrm{~m}]$ \\
Lower section & $39.69-42.69[\mathrm{~m}]$ & - & $0.30[\mathrm{~m}]$ \\
\hline \hline
\end{tabular}



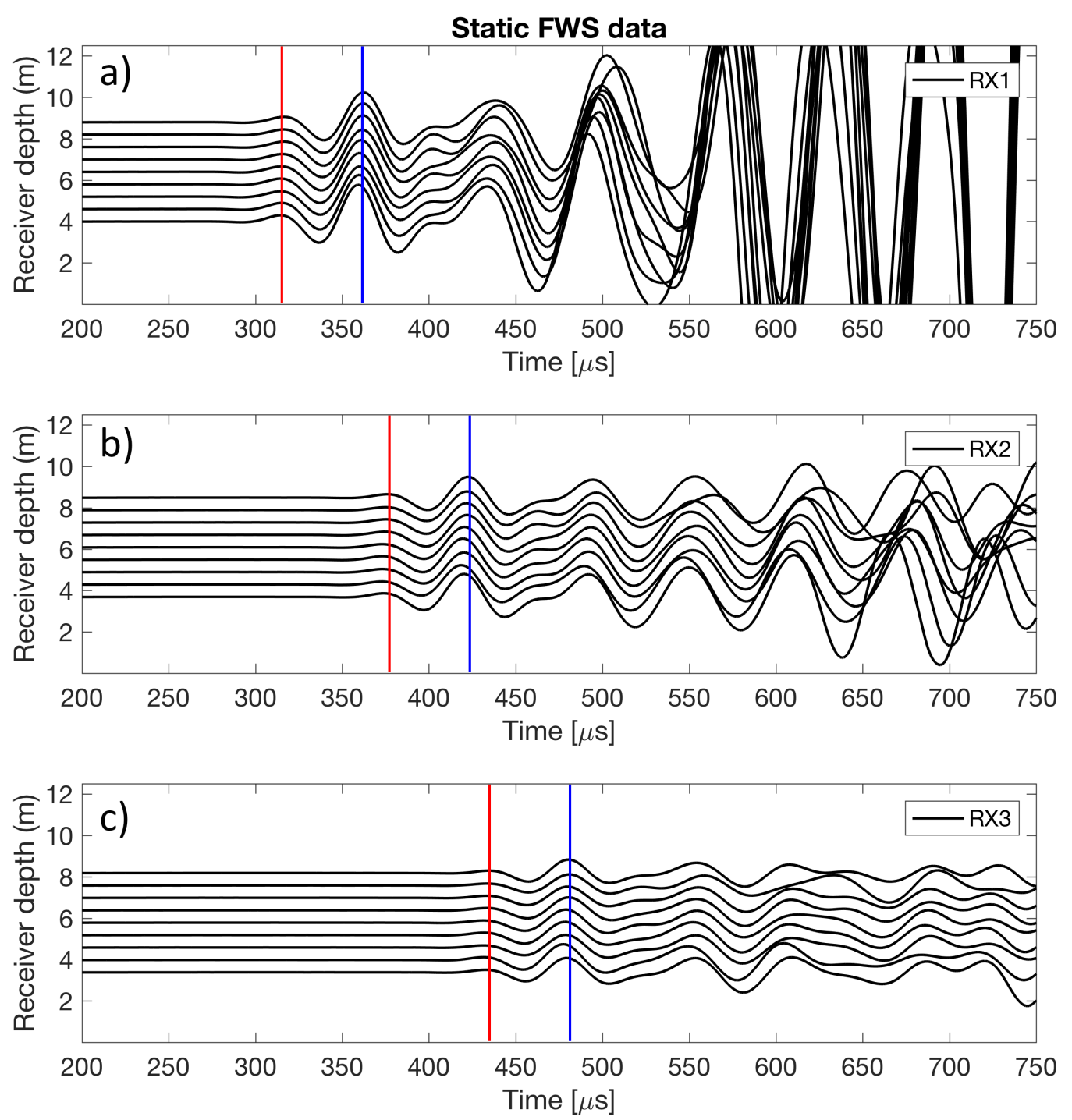

Figure 2. Static FWS data recorded in the upper section of the borehole for receivers (a) Rx1, (b) Rx2, and (c) Rx3. The offset to the source of the first receiver corresponds to the short tool configuration. The red and blue vertical lines illustrate the central time of the time windows employed to isolate one and two cycles of the first P-wave arrival, respectively. 

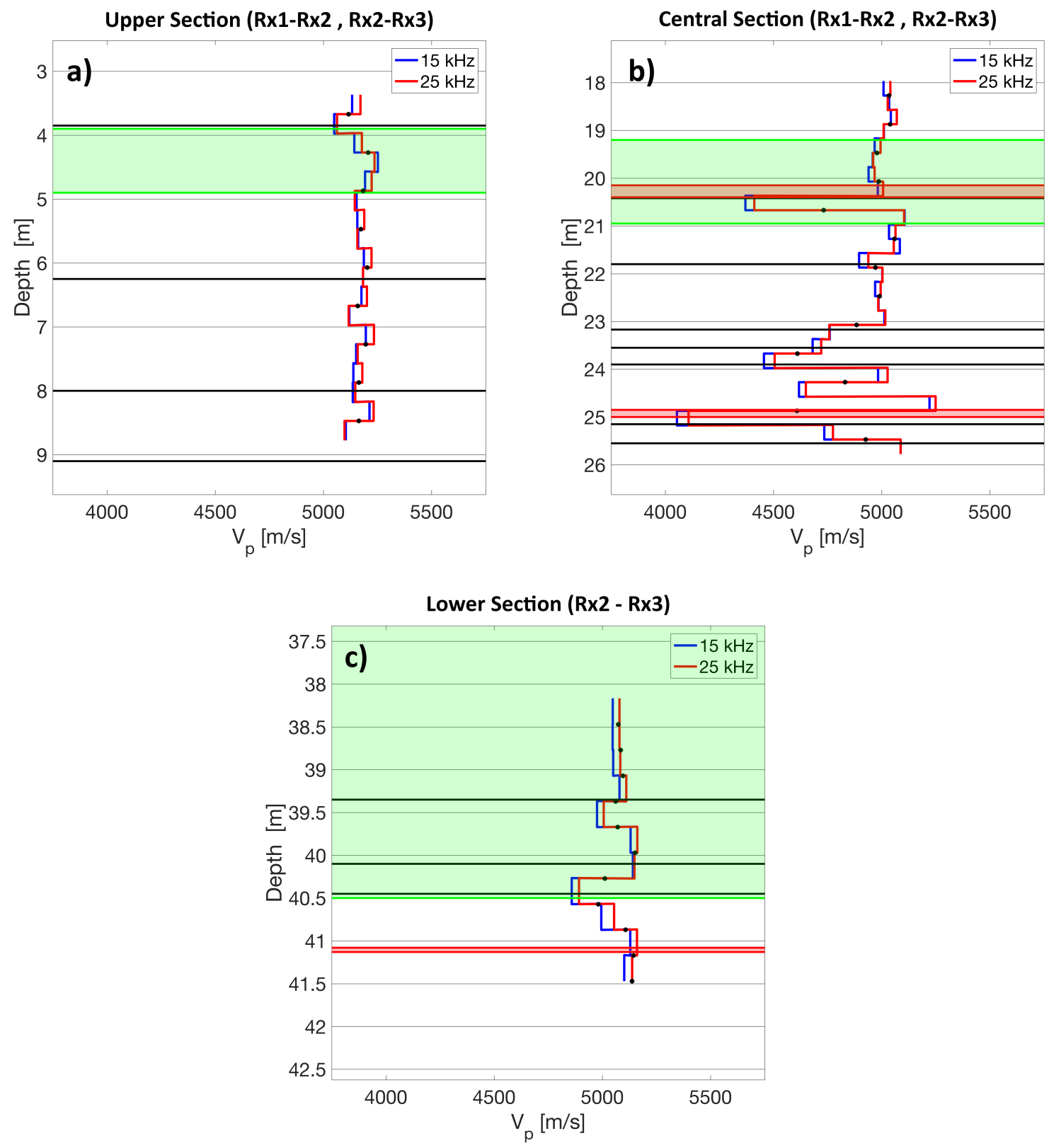

Figure 3. $\quad \mathrm{P}$-wave velocity computed for the nominal source frequencies $15 \mathrm{kHz}$ and 25 $\mathrm{kHz}$ in the upper (a), central (b), and lower (c) sections of the borehole. Regions colored in green correspond to shear zones. Black lines and red layers correspond to fractures and dykes identified in televiewer images, respectively. Dots illustrate the interval velocity between first and third receivers at the corresponding mid-point. 
Table 2. Summary of measurements performed by Wenning et al. [2018] to characterize the granodiorite host rock.

\begin{tabular}{lcc}
\hline Measurement & Parallel to foliation & Perpendicular to foliation \\
\hline P-wave velocity $V_{p}$ & $5500 \mathrm{~m} \mathrm{~s}^{-1}$ & $5100 \mathrm{~m} \mathrm{~s}^{-1}$ \\
S-wave velocity $V_{s}$ & $3430 \mathrm{~m} \mathrm{~s}^{-1}$ & $3280 \mathrm{~m} \mathrm{~s}^{-1}$ \\
Permeability $\kappa$ & $0.85 \mu \mathrm{D}$ & $0.42 \mu \mathrm{D}$ \\
Porosity $\phi$ & $<1 \%$ & $<1 \%$ \\
\hline \hline
\end{tabular}

Table 3. Physical properties of the granodiorite host rock.

Physical parameter Case 1 Case 2

Dry frame bulk modulus $K_{m} \quad 40 \mathrm{GPa} 33 \mathrm{GPa}$

Dry frame shear modulus $\mu_{m} 32 \mathrm{GPa} 29 \mathrm{GPa}$

Solid grain bulk modulus $K_{s} \quad 41 \mathrm{GPa} 37 \mathrm{GPa}$ 

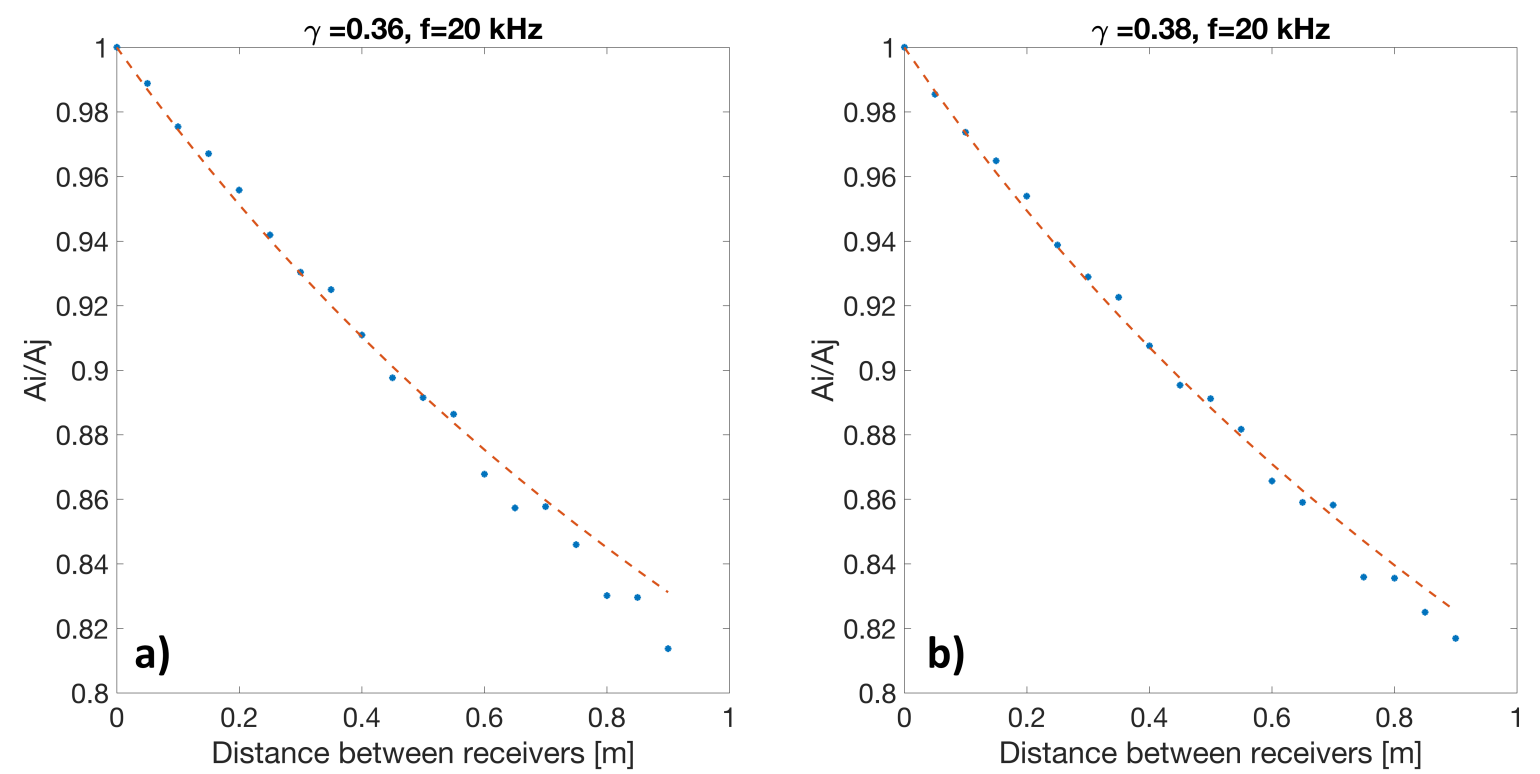

Figure 4. Geometrical spreading exponent $\gamma$ computed from spectral ratios at $f=20$ $\mathrm{kHz}$ obtained using numerical simulations of wave propagation (dots) for cases 1 (a) and 2 (b) in Table 3. The dashed curve shows the spectral ratios obtained with Eq. 6 using the $\gamma$-value indicated in plot. 

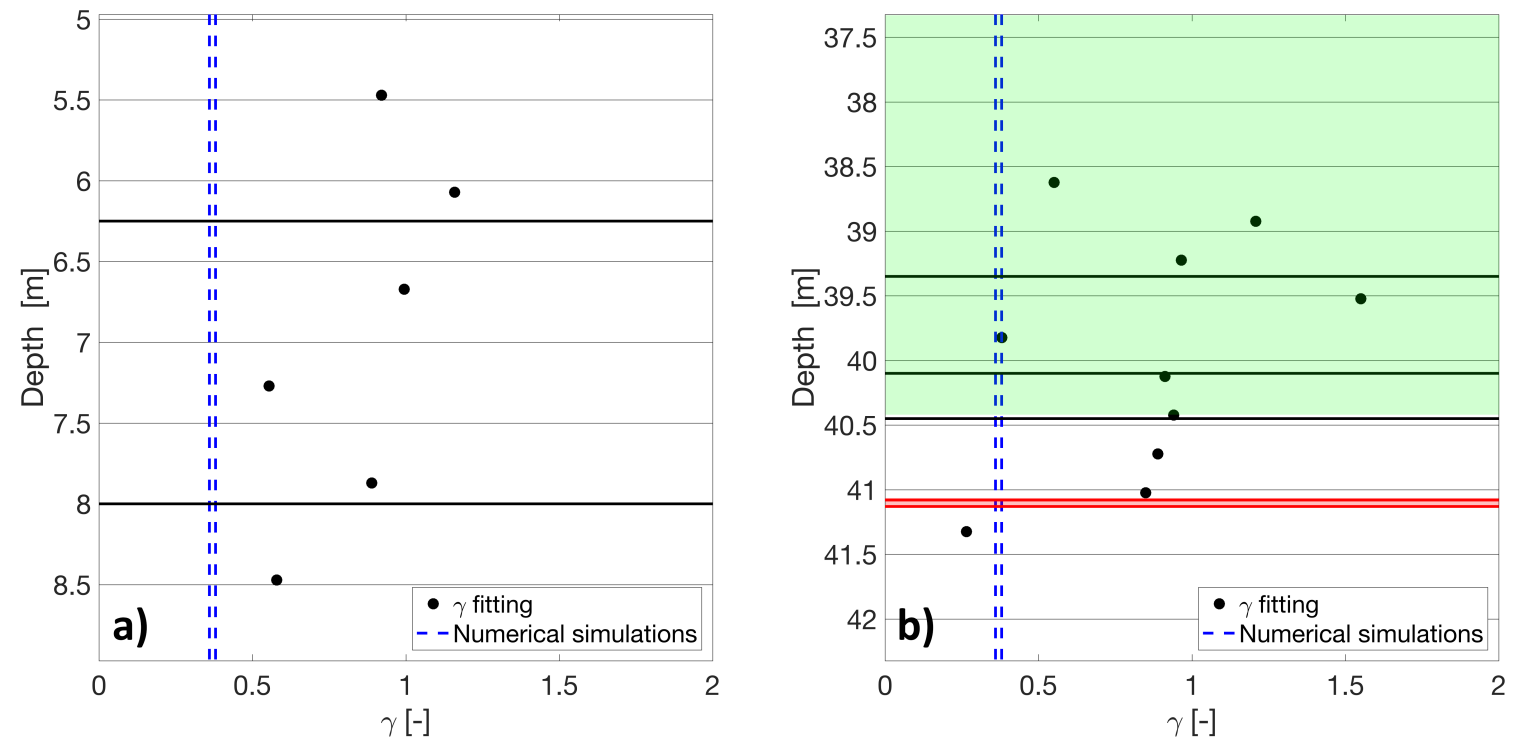

Figure 5. Geometrical spreading exponent $\gamma$ computed for the upper (a), and lower (b) sections. The depth of the dots indicates the mid point of the interval between two corresponding receivers. The blue dashed lines show the range of values of $\gamma$ computed using the numerical borehole model. Regions colored in green correspond to shear zones. Black lines and red layers correspond to fractures and dykes, respectively. 

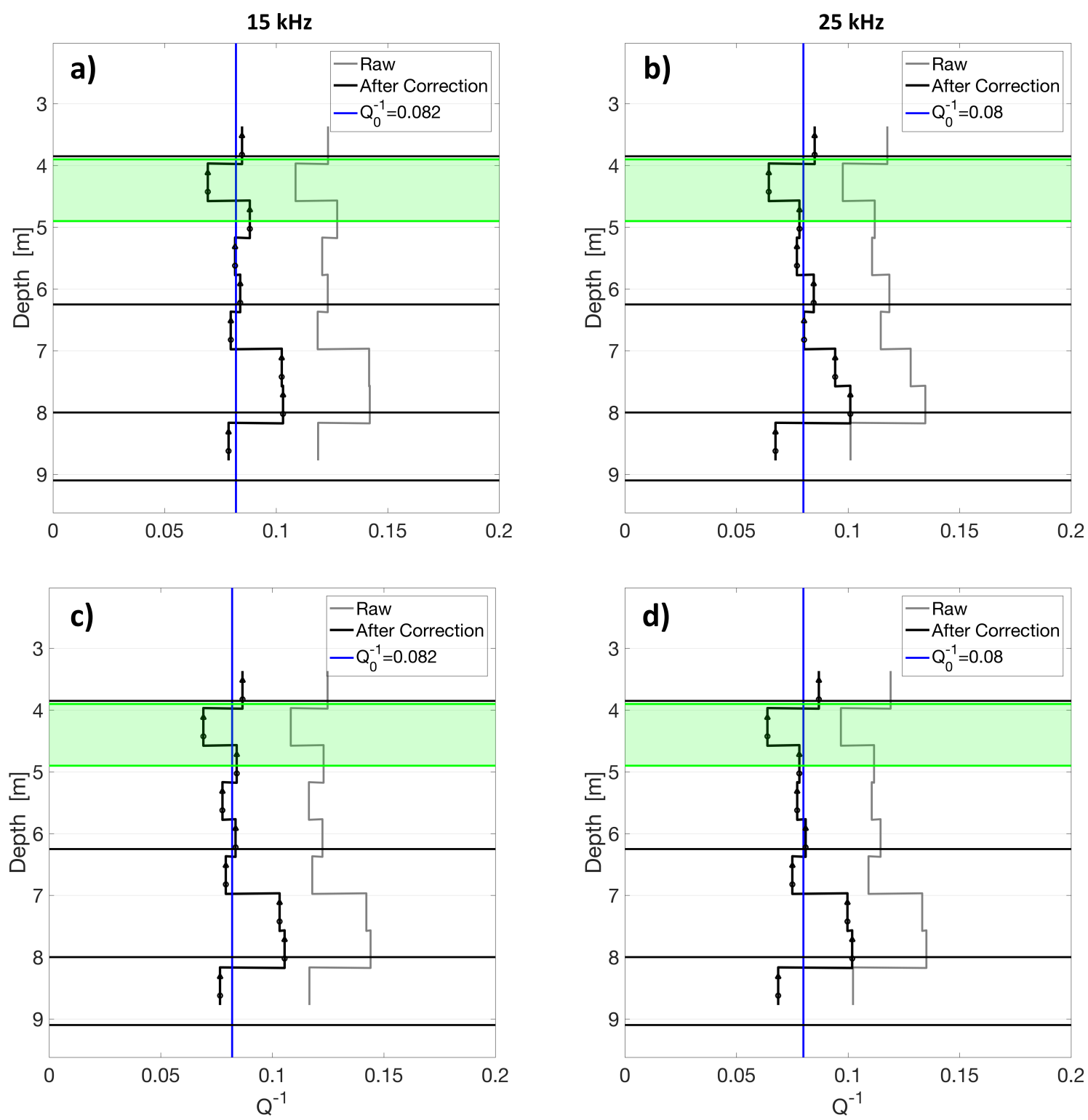

Figure 6. Attenuation as a function of depth in the upper section computed from measurements corresponding to nominal source frequencies of (a, c) 15 and (b, d) 25 $\mathrm{kHz}$ considering ( $\mathrm{a}, \mathrm{b})$ one- and $(\mathrm{c}, \mathrm{d})$ two-cycle window lengths for the isolation of the first-arriving P-wave. Black and grey solid curves correspond to attenuation estimates with and without geometrical spreading correction, respectively. The blue vertical line illustrates a mean background intrinsic attenuation $Q_{0}^{-1}$. Horizontal black lines and green zones correspond to fractures and shear zones, respectively. 

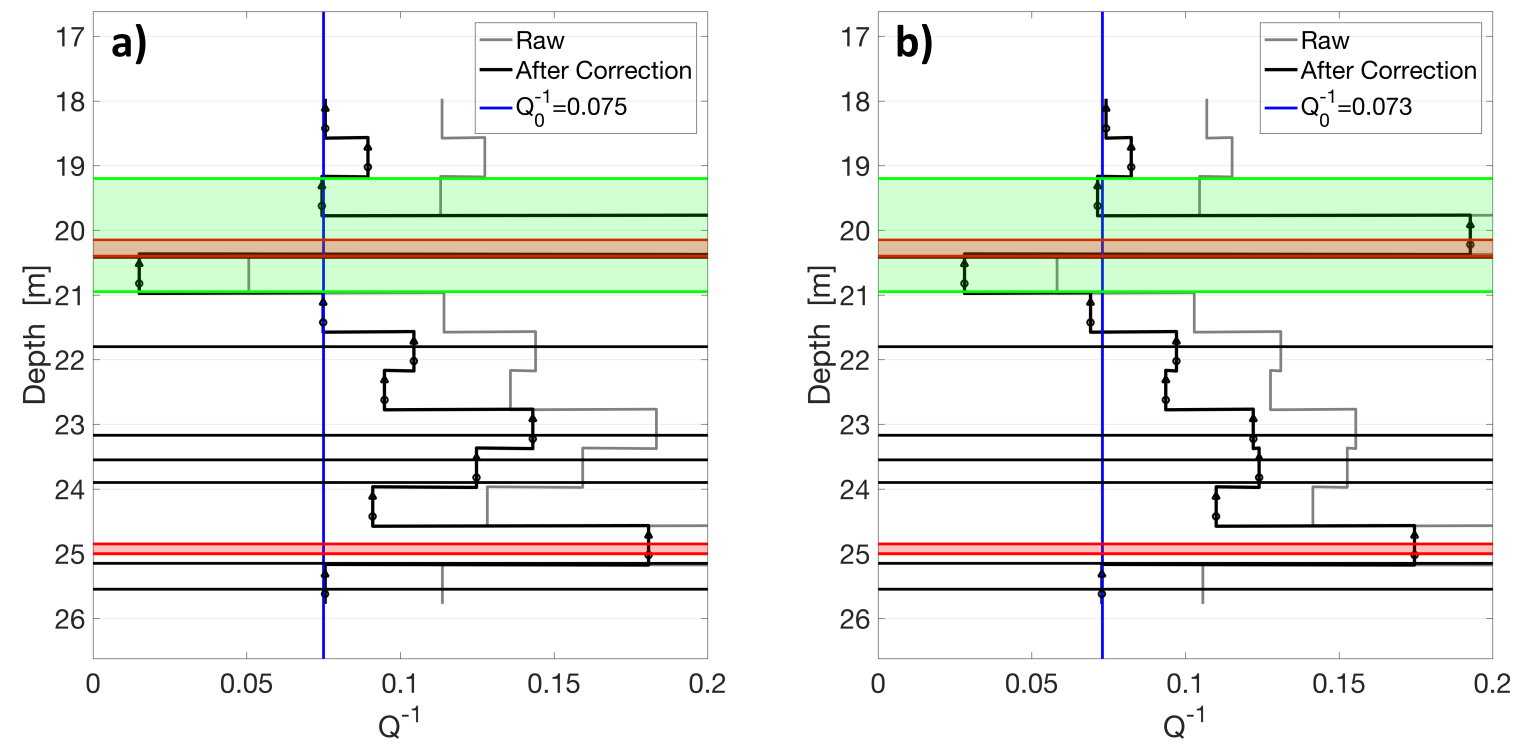

Figure 7. Attenuation as a function of depth in the central section computed from measurements corresponding to nominal source frequencies of (a) 15 and (b) $25 \mathrm{kHz}$. Black and grey solid curves correspond to attenuation estimates with and without geometrical spreading correction, respectively. The blue vertical line illustrates a mean background intrinsic attenuation $Q_{0}^{-1}$. The green zone corresponds to the shear zone. Horizontal black lines and red layers correspond to fractures and dykes, respectively, identified from televiewer images. 

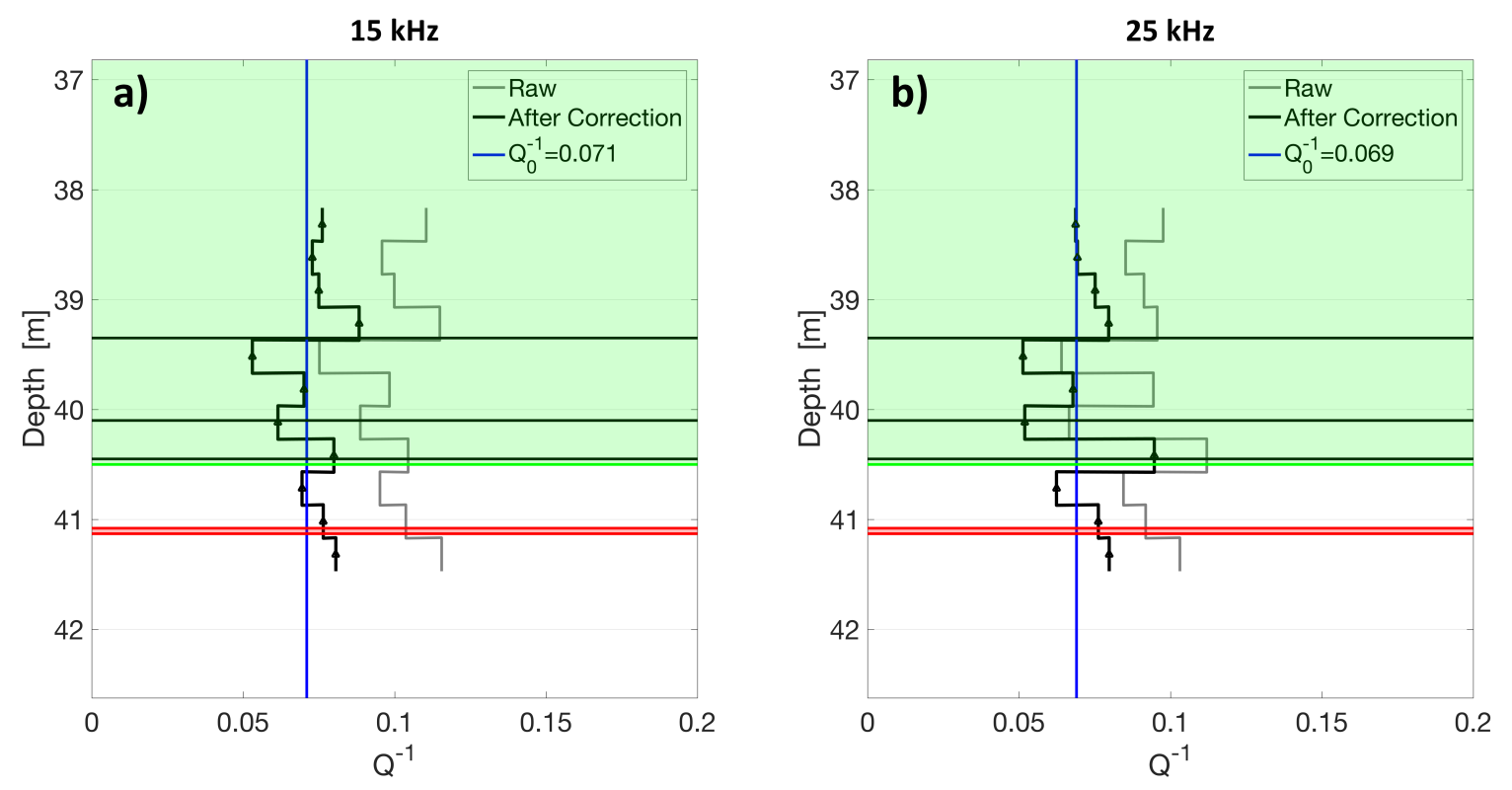

Figure 8. Attenuation as a function of depth in the lower section computed from measurements corresponding to nominal source frequencies of (a) 15 and (b) $25 \mathrm{kHz}$. Black and grey solid curves correspond to attenuation estimates with and without geometrical spreading correction, respectively. The blue vertical line illustrates a mean background intrinsic attenuation $Q_{0}^{-1}$. The green zone corresponds to the shear zone. Horizontal black lines and red layers correspond to fractures and dykes, respectively, identified from televiewer images. 


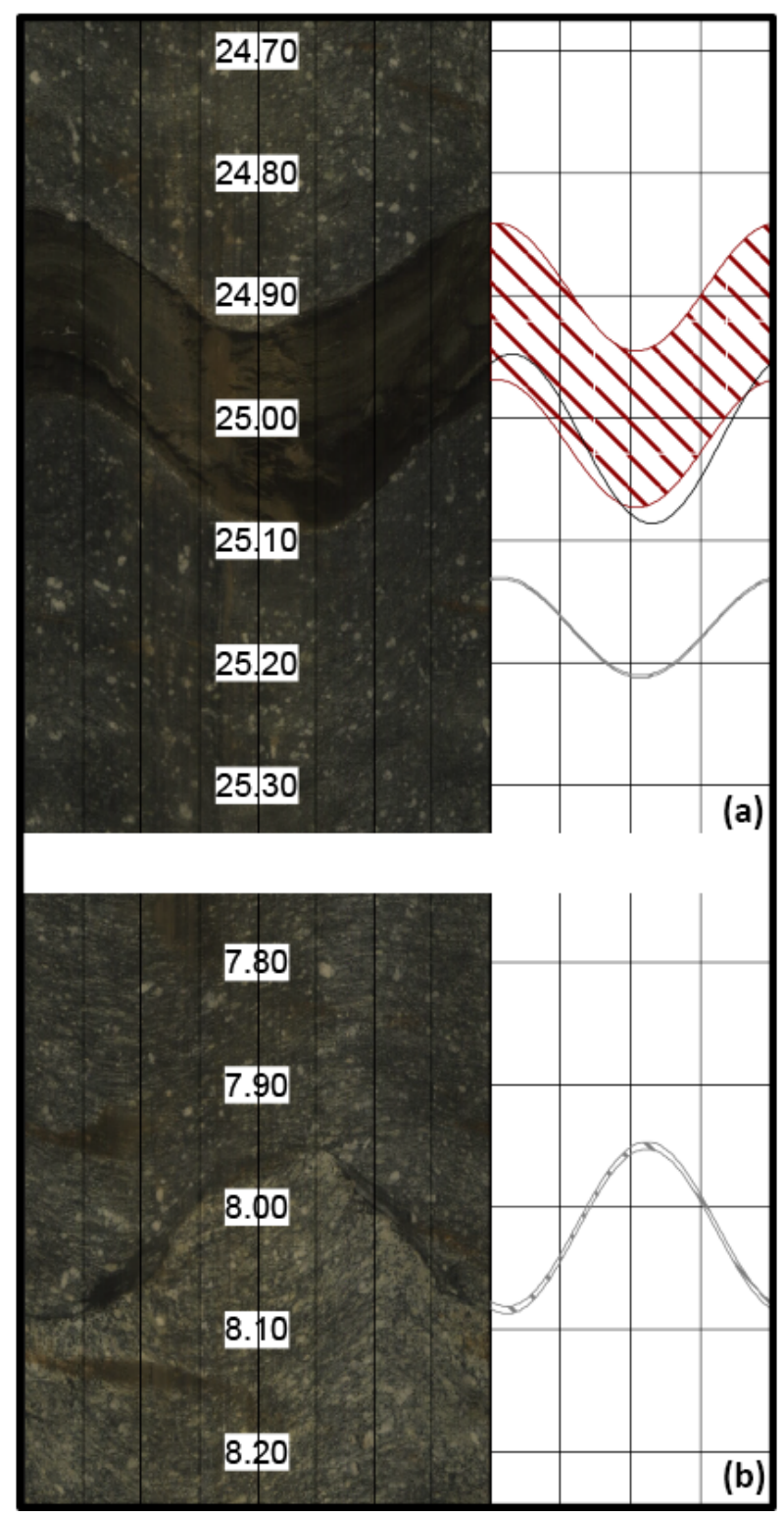

Figure 9. Televiewer images [Krietsch et al., 2018] of (a) a dyke (red layer) and (a, b) fractures (dark lines) in different sections of the borehole. 
Table 4. Transmission coefficients $T$ and fracture compliances $Z_{N}$ estimated from FWS data. Dip angles $\theta_{D}$ of the fractures with respect to the borehole trajectory were inferred from televiewer images.

\begin{tabular}{lcccc}
\hline \hline Fracture depth & $|T|$ & $\Re\left[Z_{N}\right]$ & $\Im\left[Z_{N}\right] / \Re\left[Z_{N}\right]$ & $\theta_{D}$ \\
\hline$\sim 8.0 \mathrm{~m}$ & 0.85 & $1.6 \mathrm{e}^{-13} \mathrm{~m} / \mathrm{Pa}$ & 1.2 & $50^{\circ}$ \\
$\sim 21.8 \mathrm{~m}$ & 0.78 & $3.3 \mathrm{e}^{-13} \mathrm{~m} / \mathrm{Pa}$ & 1.1 & $69^{\circ}$ \\
$\sim 23.1 \mathrm{~m}$ & 0.64 & $8.4 \mathrm{e}^{-13} \mathrm{~m} / \mathrm{Pa}$ & 0.7 & $71^{\circ}$ \\
$\sim 23.55 \mathrm{~m}$ & 0.58 & $9.9 \mathrm{e}^{-13} \mathrm{~m} / \mathrm{Pa}$ & 0.5 & $31^{\circ}$ \\
$\sim 40.40 \mathrm{~m}$ & 0.85 & $3.9 \mathrm{e}^{-13} \mathrm{~m} / \mathrm{Pa}$ & 0.4 & $37^{\circ}$ \\
\hline \hline
\end{tabular}




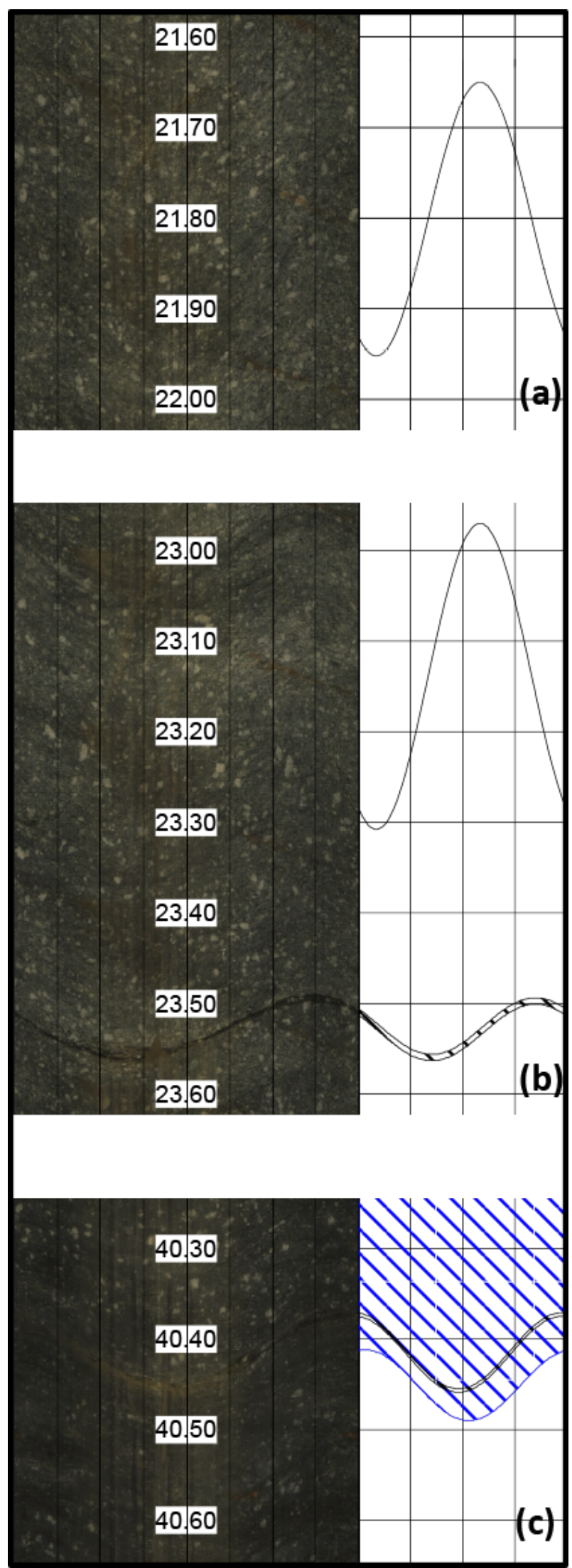

Figure 10. Televiewer image and its interpretation for the fractures in the central and lower sections given in Table 4. Shear zones are identified with diagonal blue lines. 


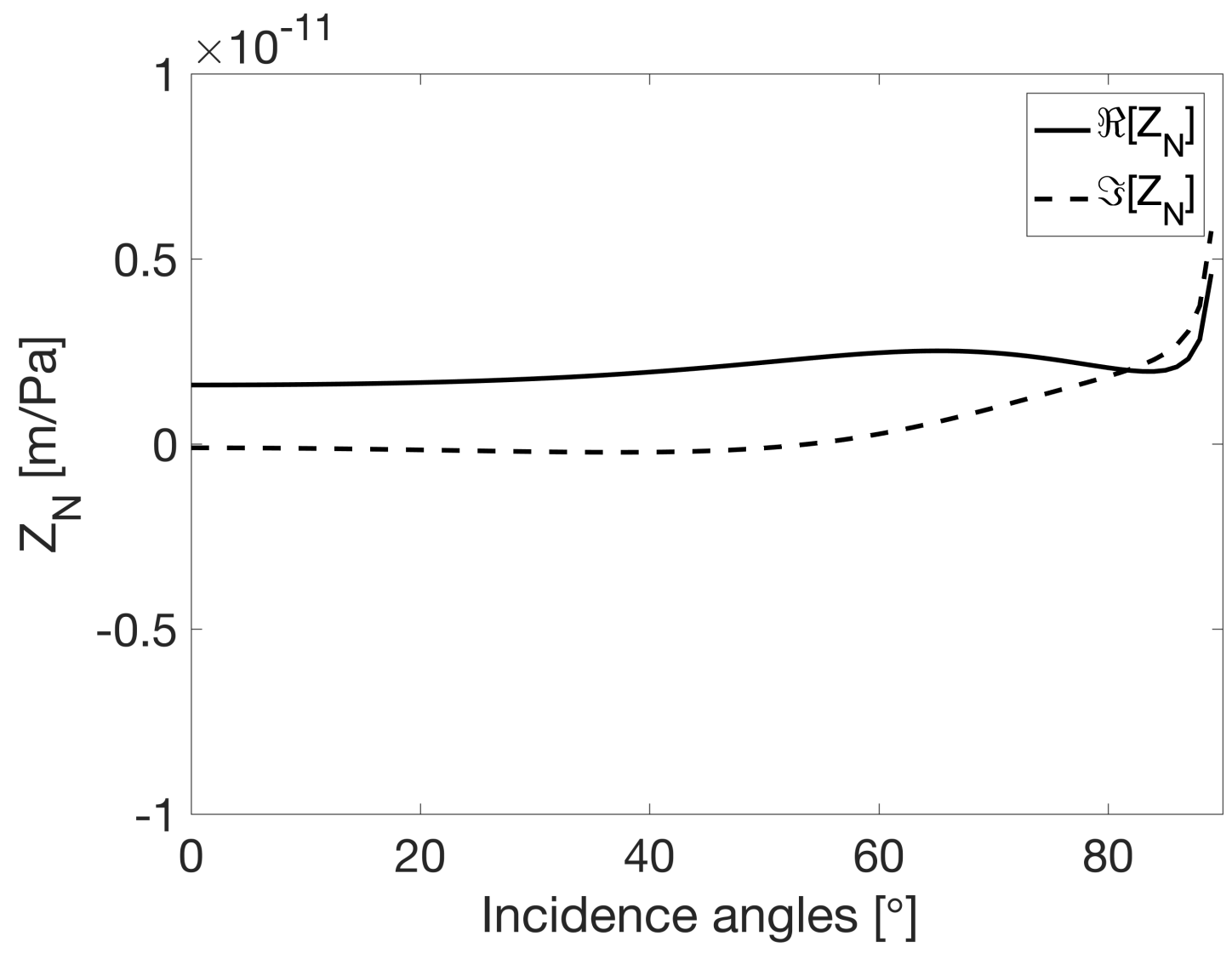

Figure 11. Real and imaginary components of the fracture compliance computed using Eq. 13 and considering the P-wave transmission coefficient at different incidence angles. 


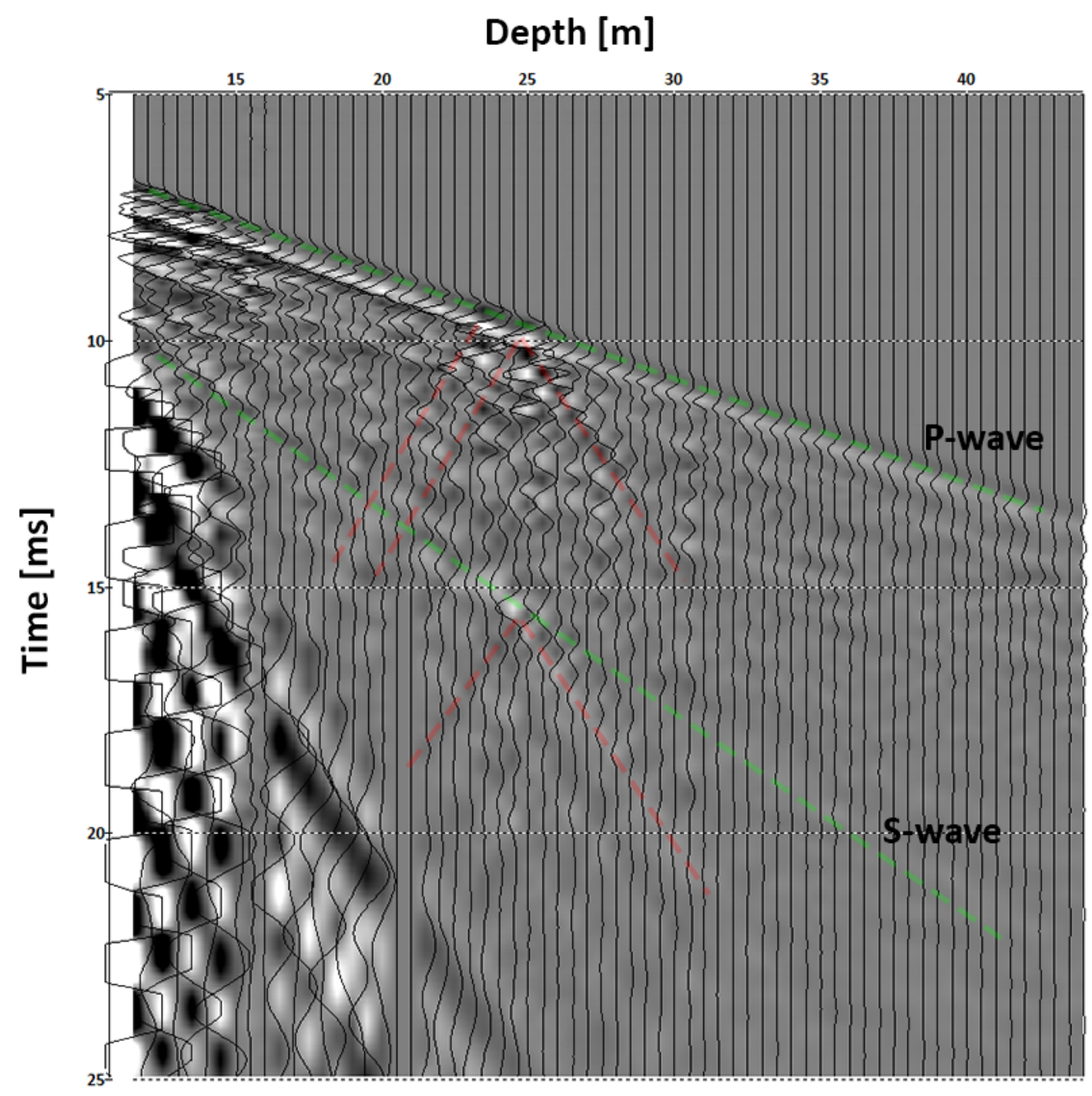

Figure 12. Zero-offset hydrophone VSP data collected with sensors located at depths ranging from 11.5 to $44 \mathrm{~m}$ along the INJ2 borehole. The green lines denote the arrivals of the $\mathrm{P}$ - and S-waves propagating along the borehole wall. Red lines correspond to the arrivals of tube wave generated at the fractures. 


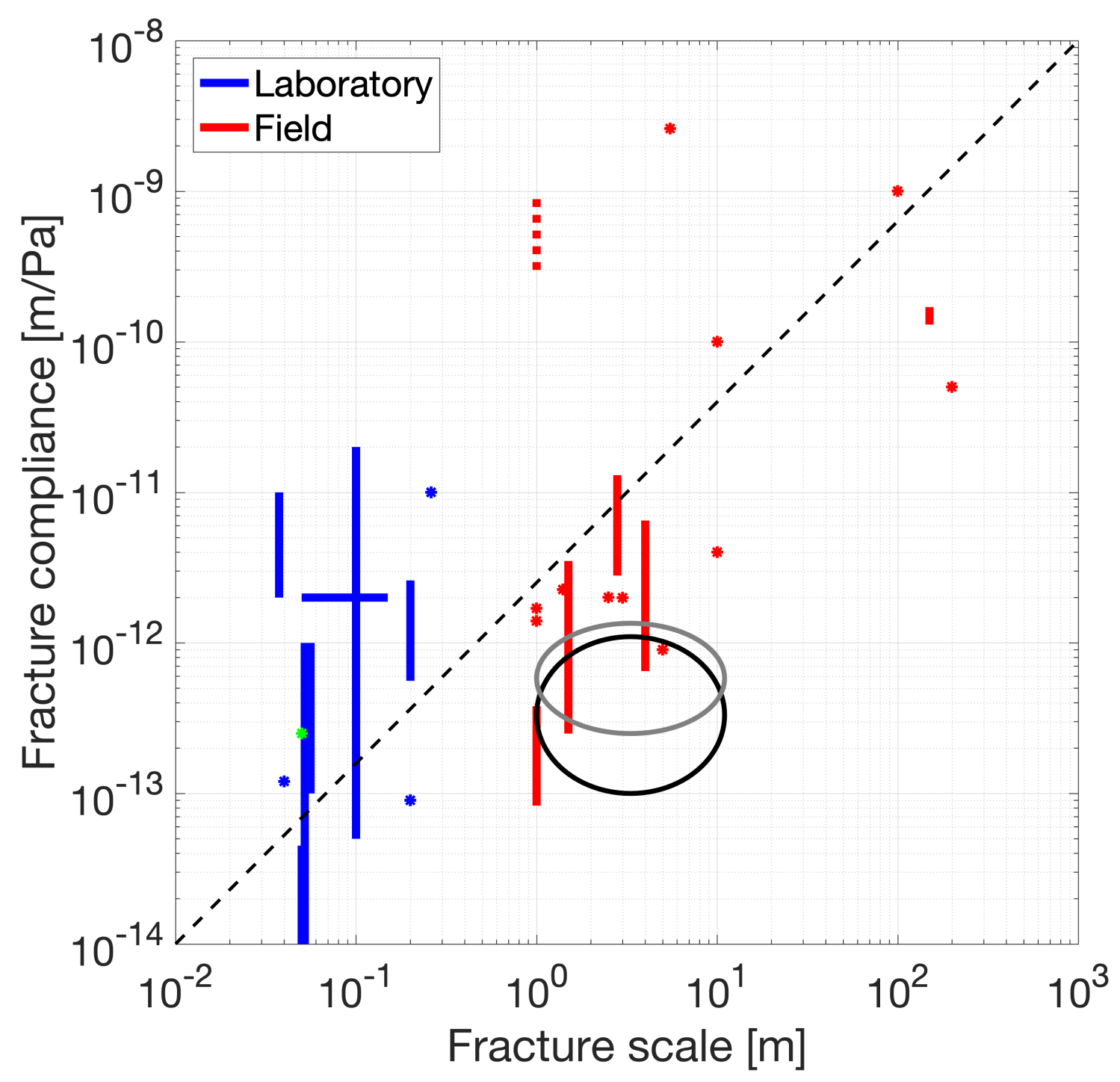

Figure 13. Static (blue) and dynamic (red) fracture compliance values as function of fracture size compiled from the literature. The black and grey ellipses indicate the range of the real component and absolute value of the compliances reported in this work, respectively. The green dot corresponds to the compliance estimated from the laboratory measurements on synthetic samples by Rathore et al. [1995]. 

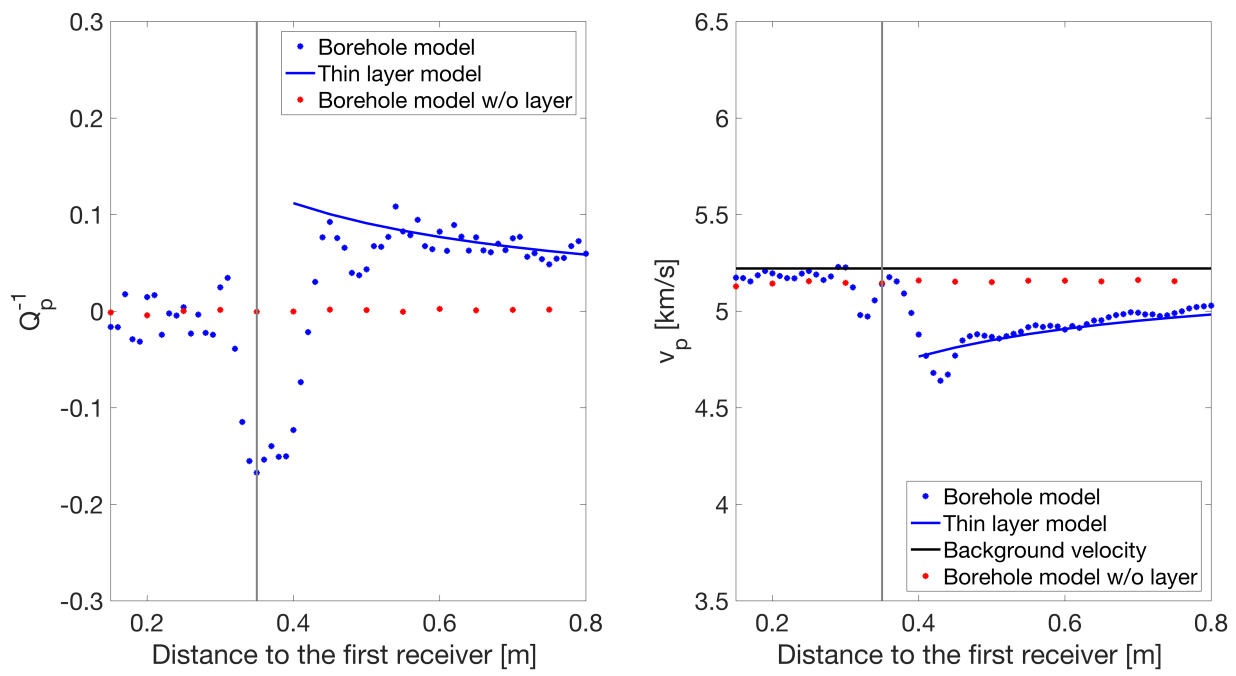

Figure 14. Effective attenuation and velocity as functions of the distance to the first receiver for a frequency of $20 \mathrm{kHz}$. Blue and red dots correspond to the results of numerical simulations of wave propagation in a borehole with and without a thin layer, respectively. Solid blue lines represent the results of a simpler theoretical model that performs planewave propagation across a single layer. Grey vertical line marks the position of the thin layer. Black curve in the right panel corresponds to the velocity of the background formation. 

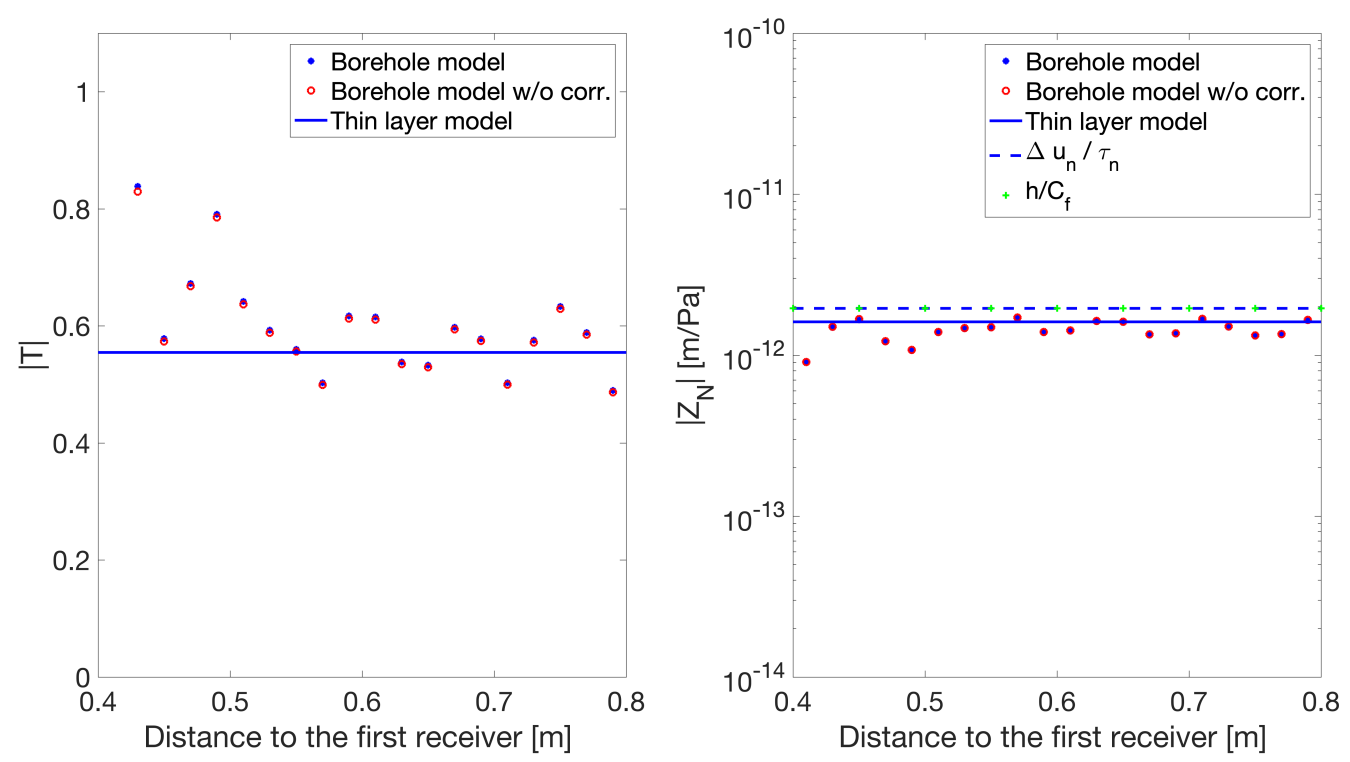

Figure 15. Magnitude of the transmission coefficient and normal compliance as functions of the distance between receivers for $20 \mathrm{kHz}$. Dots correspond to the results of the numerical simulation of wave propagation in a borehole. Solid lines represent the results of the simpler numerical model that performs plane wave propagation across a single thin layer. Dashed blue line shows the normal compliance computed from the thin-layer model following its classical definition as the ratio between the jump in normal displacement $\Delta u_{n}$ and the average normal stress $\tau_{n}$ across the fracture. Green symbols show the compliance estimated as the ratio between the fracture thickness $h$ and its undrained P-wave modulus $C_{f}$ 